https://helda.helsinki.fi

\title{
On Formalism Freeness : Implementing Gödel's 1946 Princeton Bicentennial Lecture
}

Kennedy, Juliette

2013-09

Kennedy , J 2013 , ' On Formalism Freeness : Implementing Gödel's 1946 Princeton

Bicentennial Lecture ' , Bulletin of Symbolic Logic , vol. 19 , no. 3 , pp. 351-393 . https://doi.org/10.2178/bsl.1903030

http://hdl.handle.net/10138/42538

https://doi.org/10.2178/bsl.1903030

publishedVersion

Downloaded from Helda, University of Helsinki institutional repository.

This is an electronic reprint of the original article.

This reprint may differ from the original in pagination and typographic detail.

Please cite the original version. 


\title{
ON FORMALISM FREENESS: IMPLEMENTING GÖDEL'S 1946 PRINCETON BICENTENNIAL LECTURE
}

\author{
JULIETTE KENNEDY
}

It seems in no way appropriate that Cantor's Absolute be identified with set theory formalized in standardized logic ... - Bernays, Letter to Gödel, 1961.

We don't need representations. The world is right there ... The world shows up for us.-Alva Noë, 2012.

\footnotetext{
Abstract. In this paper we isolate a notion that we call "formalism freeness" from Gödel's 1946 Princeton Bicentennial Lecture, which asks for a transfer of the Turing analysis of computability to the cases of definability and provability. We suggest an implementation of Gödel's idea in the case of definability, via versions of the constructible hierarchy based on fragments of second order logic. We also trace the notion of formalism freeness in the very wide context of developments in mathematical logic in the 20th century.
}

$\S 1$. Introduction. That mathematics is practiced in what one might call a formalism free manner has always been the case - and remains the case. Of

Received August 8, 2012.

This paper was first presented at the Winter Meeting of the Eastern Division of the American Philosophical Association in 2009. Subsequently it was presented at numerous other seminars, and I thank those audiences for their many helpful questions and comments. This paper was completed while I was a member of the School of Historical Studies of the Institute for Advanced Study during the academic year 2011-12. I thank the Otto Neugebauer Fund for this support as well as the Academy of Finland and the Väisälä Foundation for their additional support. John Baldwin, Andres Villaveces and Jouko Väänänen had an important influence on my thinking during the writing of this paper and I wish to express my gratitude to them for extensive correspondence and discussions. I also wish to thank Andrew Arana, Patricia Blanchette, John Burgess, Mic Detlefsen, Juliet Floyd, Curtis Franks, Wilfrid Hodges, Tapani Hyttinen, Meeri Kesälä, Penelope Maddy, Menachem Magidor, Saharon Shelah, and Scott Weinstein for helpful discussions and correspondence, as well as two anonymous referees, whose comments and corrections lead to important improvements in the paper.

(c) 2013, Association for Symbolic Logic $1943-5894 / 13 / 1903-0003 / \$ 5.30$ DOI:10.2178/bsl. 1903030 
course no one would have thought to put it this way prior to the emergence of formal systems in the late nineteenth and early twentieth centuries, ${ }^{1}$ what is interesting, to the foundationalist at least, is that mathematicians continue to practice their subject in a formalism free manner even now, in what one might call the post-foundational era.

What was the foundational era? This was the period inaugurated, roughly, by Frege and continuing through the first part of the twentieth century, ${ }^{2}$ during which worries essentially about consistency - to simplify matters only a little-motivated the development of various foundational formal systems; a development which, if it did not exactly set those worries to rest, at least increased confidence in the unlikelihood of their ever being realized. The foundationalist objective which eventually emerged was stated in a preliminary but exact form ${ }^{3}$ by Hilbert and his school. In its full form what we are calling the formalism-oriented foundationalist program, was simply this: embed mathematics in a formal language with an exact proof concept and an exact semantics, such that the proof concept is sound and complete with respect to the associated semantics as well as syntactically complete in the sense that all propositions that can be written in the formalism are also decided. The preservation of meaning, as well as other epistemically valuable features, were important desiderata which were to be shown in various ways. Characteristic of the Hilbert Program was the demand that the formal environment, however it was conceived, be finitary; though what the Hilbert School meant by the term "finitary" was not clear at the time, and indeed would not be resolved, at least to a reasonable degree of satisfaction, until Tait's 1988 [79]. The principal demand imposed by the Hilbert Program of course, was that the formalism be demonstrably, indeed internally consistent - a demand which, if met, would have assuaged qualms about the use of infinitary concepts, along with, or more precisely by means of, resolving the consistency issue. The demand for an internal consistency proof was replaced after Gödel's 1931 Incompleteness Theorems with a multiplicity of coping mechanisms - or assertions to the effect that such were not needed, as the case may be.

Setting aside the interesting mathematical questions - indeed the entirely new subject areas altogether, ${ }^{4}$ which emerged from the various foundationalist programs of the time, it is simply a fact that foundationalism in this form came and went with little lasting impact on mathematical practice.

\footnotetext{
${ }^{1}$ I.e., propositional and first and second order predicate logic, Peano Arithmetic, ZermeloFrankel set theory, and the various intuitionistic systems etc. Some of these systems were attached to foundational programs. In that case we refer to them as "foundational formal systems."

${ }^{2}$ We do not offer an end date, though many would.

${ }^{3}$ A so-called "naturalized version," according to Curtis Franks in his [26], rather than a project in foundational epistemology.

${ }^{4}$ i.e., set theory, model theory, proof theory, etc.
} 
The demonstrable failure of syntactic completeness for systems like Peano Arithmetic (via Gödel's First Incompleteness Theorem) and the attendant collapse of the Hilbert Program (via the two Incompleteness Theorems together) affected the mathematician's working life very minimally, if at all. The reasons for this are interesting, and though they are not strictly speaking the subject of this paper, we can take note of certain responses at critical moments. For Kreisel, a sine qua non of interest in the Hilbert Program was not the consistency question per se but rather the presumed equivalence of second order consequence with, "at least in suitable contexts," formal derivability (as had been shown in the first order case). ${ }^{5}$ However the question is of secondary importance for mathematics, concerned as mathematics is with (in Kreisel's terminology) fundamental analysis. "Logical hygiene," as he called it, might be useful, e.g., psychologically; but independence proofs, formalization, and such like, are not what mathematics deals with at its most fundamental level. "C'est magnifique, mais ce ne sont pas les fondements"6 he would say, of formalization.

From a completely different standpoint, Gödel echoed the thought that mathematicians and logicians are involved in two distinct enterprises. About his own Incompleteness Theorems, although they had a negative impact on e.g., the Hilbert program, ${ }^{7}$ in the most important conceivable sense, ${ }^{8}$ they should have no impact on the working life of the average mathematician (or set theorist) otherwise: "As to problems with the answer Yes or No, the conviction that they are always decidable remains untouched by these results." 9 In other words, a way has to be found to set incompleteness to the side-with conviction.

This paper is an attempt to arrange a differently interested encounter with the concept of formalization, in the light of the tendency in mathematical logic over the last century to bend away from foundational epistemology and towards the subject of mathematics itself, and the project, simply put, of understanding it more fully. Expressing an attitude toward formalization which is opportunistic rather than foundational, in that very simple sense the contemporary model theorist, for example, is, at best, a local foundationalist - wanting to shed light on a certain area of the practice, but eschewing any attempt to supply a global foundation for it.

Our starting point is Gödel's Completeness Theorem of 1929, showing that first order consequence admits a semantic characterization. ${ }^{10}$

\footnotetext{
${ }^{5}$ See [53], p. 146.

${ }^{6}$ Kreisel, op cit.

${ }^{7}$ in Gödel's view. But see Feferman's [23], Detlefsen's [18], and Franks's [26] on intensional adequacy and the Second Incompleteness Theorem.

${ }^{8}$ namely decidability.

${ }^{9}[193$ ?] in [35].

${ }^{10}$ It is interesting to note that this way of viewing the theorem did not appear in print until Robinson's 1951 [71].
} 
Curtis Franks sees Gödel's Completeness Theorem as entirely definitive and world-changing, precisely because that theorem displaced certain philosophically loaded views of logical consequence prevalent at the time. ${ }^{11}$ Gödel's theorem revealed a correspondence between a fundamental syntactic notion, proof, and a fundamental semantic notion, truth. Depending on their philosophical agenda, logicians could now restrict themselves wholly to syntax, or on the other hand wholly to semantics, without risk, as it were, of leaving out the other point of view. Franks's important observation is that the redesign of logical terrain achieved by that theorem was so comprehensive and so convincing, that by now the historical enterprise, the going back in time in search of an understanding of what e.g., Bolzano's notion of logical consequence would have been, or even Frege's, constitutes hard labor for the twenty-first century logician. Franks writes:

Gödel never considered that others' ... inability to see their way to the completeness theorem derived from their focus being held elsewhere. But thinking about logic in the terms that defined Gödel's contribution was not universal, perhaps not even common, in the early twentieth century. His early writing plays a major part in an implicit argument that the correspondence of proof and truth, of logical form and content, is a proper way of thinking about logical completeness. While the theorem contained in Gödel's thesis is a cornerstone of modern logic, its far more sweeping and significant impact is the fact that, through its position in a network of technical results and applications, the way of thinking underlying the result has come to seem definitive and necessary, to the extent that we have managed to forget that it has not always been with us.

A truism of our Gödelian inheritance is that the syntax/semantics distinction is clearly defined. The view taken here is that that particular logical terrain has since turned out to be so intricate and fine-structured; so replete with delicate entanglements of syntax and semantics on the one hand, as well as with what appear to be purely semantic phenomena on the other, that it is nearly not a distinction anymore at all — being virtually eradicated by such entanglements.

We attempt to survey this disordered terrain. Our point, simply put, is this: while the syntax/semantics distinction is still very much with us, so is formalism freeness. For Brouwer the radical decoupling of mathematics with language and its grounding in time intuition, was its fundamental feature.

\footnotetext{
${ }^{11}$ About Bolzano, for example, Franks writes: “ $\ldots . \ldots$ in the end, it was the objective grounding of truths which drove him ... Modern logicians, by contrast, have no expectation that their craft will uncover ultimate grounds. Many do not even believe in such things. What remains of Bolzano's intricate scheme for writers who do not share his metaphysical aspirations?" See [27]. Frege's is another important example of a philsophically freighted notion of logical consequence.
} 
We read Gödel's 1946 lecture as an important but perhaps overlooked step in this line of thought, not with respect to language necessarily, or to time intuition, but with respect to formalization altogether; in particular we will interpret Gödel there as making the suggestion, albeit in a preliminary form, that Turing's analysis ${ }^{12}$ of the notion of computability be replicated, not for the notion of computable function but for the concept of definabilitywitnessing its formalism independence, as it were. We will sketch at the end of this paper an implementation of this, based on our interpretation of that lecture.

§2. Foundationalism: Initial thoughts. We begin with the following observation. If we shift from standard mathematical practice to the more restricted area of research in mathematical logic, we can notice a shift within certain areas in the latter, in the form of a movement away from the leading role of formal languages. In fact, this tendency towards the avoidance of a formal language or logic, if not indeed of formalism-oriented foundationalism altogether, has been underway in various subspecialties for some time. On the model-theoretic side this developed partly from motivations like the desire to find a general and natural framework for results such as Morley's Theorem, ${ }^{13}$ the desire to work in a framework without compactness, ${ }^{14}$ or the idea that definable sets of reals (just as definable sets of any o-minimal structures) can be viewed in a natural way as elements in a sequence of boolean algebras closed under projection mappings and similar mathematical properties, an entirely logic free notion of definability. ${ }^{15}$ Whatever the reason, it can reasonably be said that by now certain branches of model theory have developed a plethora of what one might call purely semantic methods, ${ }^{16}$ a phrase we will attempt to define more carefully below. The following remark of Saharon Shelah, describing the thesis of his [75, 76], is in effect a description of (one aspect of) this development: "Considering classical model theory as a tower, the lower floors disappear-compactness, formulas, etc. ... the higher floors do not have formulas or anything syntactical at all." 17

We consider particular instances of this vast development of semantic methods - a species of what we are calling formalism freeness ${ }^{18}$ - and elaboration of them from the time of the Completeness Theorem, below. What

\footnotetext{
${ }^{12}$ see below.

${ }^{13}$ We are referring here to Shelah's [75, 76]. Morley's Theorem says in first order logic that a countable theory that is categorical in one uncountable cardinality is categorical in every uncountable cardinality.

${ }^{14}$ See [5] and [6].

${ }^{15}$ See Shelah's [75]; see also Pillay and Steinhorn's [69].

${ }^{16}$ The term "pure semantic method" is due to Baldwin, [3].

${ }^{17}$ Shelah, personal communication.

${ }^{18}$ For the definition of this term see section 3 below.
} 
interests us is the semantic reformulation of logical theorems - a development originating, so to speak genetically, in Gödel's 1929 Completeness Theorem and "spiritually" much earlier, in the work of Peirce, Schröder and others. Formalism freeness is not an all or nothing affair, but a matter of degree; a tendency of thought which was realized here and there, sometimes very explicitly, and sometimes in a very disguised form and taking place only in certain areas.

A link between model theory as it has been traditionally pursued, and certain other lines of thinking, might be worth pointing out. The tendency to suppress various aspects of a formalism - the development in logic which inspires us here- has been accompanied on the philosophical side of things, particularly recently, by a sense of fatigue with a priorist or "first" philosophical analysis, to use Maddy's terminology, and the forefronting of philosophical naturalism, ${ }^{19}$ or implicitly naturalistic approaches. ${ }^{20}$ Naturalism, in particular the naturalist's recommendation to "track mathematical practice," advocates a withdrawal of the foundationalist critique as its very starting point. ${ }^{21}$

The naturalist's suggestion to become absorbed in mathematical practice bifurcates along various lines - one can become absorbed in different ways, after all. Maddy's second philosopher ${ }^{22}$ is bound by a particular view of mathematics' empirical applications, for example, which the methodological naturalist is, generally, not. Cutting across all of these lines of thinking is a shared belief in the obsolescence of the foundational project. Franks puts it this way in his [26]:

Recent philosophical writing about mathematics has largely abandoned the a priorist tradition and its accompanying interest in grounding mathematical activity. The foundational schools of the early twentieth century are now treated more like historical attractions than like viable ways to enrich our understanding of mathematics. This shift in attitudes has resulted not so much from a piecemeal refutation of the various foundational programs, but from the gradual erosion of interest in laying foundations, from our culture's disenchantment with the idea that a philosophical grounding may put mathematical activity in plainer view, make more evident its rationality, or explain its ability to generate a special sort of knowledge about the world. ${ }^{23}$

\footnotetext{
${ }^{19}$ See [61, 60, 62], [26].

${ }^{20}$ See e.g., [2], [19] and [3].

${ }^{21}$ Of the myriad versions of naturalism, we are thinking of, roughly, methodological naturalism as defined in Weir, [74]. As the view specializes to mathematics proper we look to [26] and to some extent $[61,60]$, though Maddy doesn't entirely reject the idea of set-theoretic foundations, in a somewhat limited sense.

${ }^{22}$ See Maddy, ibid.

${ }^{23}$ [26] p. 169.
} 
The question whether the naturalist's loss of interest in foundational programs is linked in any substantive way with the various sensibilities driving the development of the particular language free developments which interest us here - though an aversion to global foundational programs has been in place particularly in model theory for much longer ${ }^{24}$ - will not be considered any further, as this would take us both too far afield, and into a mode of analysis at odds with that we wish to pursue here. We return to naturalism in our concluding remarks.

§3. What is formalism freeness? When Gödel proved his Incompleteness Theorems he left open what an effectively given formal system means. Only after Turing's fully mathematical definition of effective computability was given, was Gödel ready to declare the concept of a formal system clearly defined. In this paper we have a more liberal notion of a formal system, or formalism, as we call it.

By a formalism, or a logic, we mean a combination of a list of symbols, commonly called a signature, or vocabulary; rules for building terms and formulas, a list of axioms, ${ }^{25}$ a list of rules of proof, and finally a definition of the associated semantics. We leave it ambiguous whether we mean axiom systems, such as Peano arithmetic and ZFC set theory, or formal languages such as first and second order logics. This intentional ambiguity is manifest in the case of second order logic.

Some question whether a logic needs a semantics at all, or regard semantics as an after-thought, a possibly helpful but otherwise inessential addition to the logic. This is not the concept of logic we use. In this paper the semantics is in the main role and the other aspects of a formalism are subordinate to it. By and large we assume that the various lists and rules that are constituent of a logic are effectively given. In such a case we call the formalism finitary. We consider also infinitary formalisms, such as the infinitary languages $L_{\kappa \lambda}$.

With this concept of formalism we associate formalism freeness with the suppression of any or all of the above aspects of a logic, except semantics. The position taken in this paper is that the associated semantics cannot be suppressed. Of course vocabulary in the informal, natural language sense, that is, as detached from any formalism based on it, is always a residue of the practice and in that sense is not suppressed in the model-theoretic applications we are considering here (or for that matter in mathematical investigations of any kind). Our distinction here is between the natural

\footnotetext{
${ }^{24}$ If not from the very beginning. E.g., Maltsev, who was the first to prove the Compactness Theorem in full generality, thought of logic as part of algebra; and the work of Robinson and Tarski in the 1950s is largely, if not wholly unconnected to global foundational programs.

${ }^{25}$ Vocabulary considered together with rules for building terms as well as deduction rules are sometimes referred to as the syntax.
} 
language concept of a vocabulary, and vocabulary as a constituent of a formal language. ${ }^{26}$

We immediately address the obvious criticism: what is our knowledge of semantics based on if not on some formalism? The answer to this question is the heart of our approach. We take it as given that mathematics has-and has always had-subject matter, and the question is, whether and to what extent the various relevant formalisms capture it. ${ }^{27}$

In (the informal version of) axiomatic set theory for example, what is emphasized is "the intuitive notion of the cumulative type structure," as Kreisel puts it in his [53, p. 144], over the formal theory ZFC. Gödel did not formulate his position in the way we put it here, but we will argue in this paper that various of the notions of formalism freeness that we will isolate can be grounded in the ambient, framing philosophical territory within which and pursuant to which Gödel tended his specific mathematical projects.

3.1. Language, faithfulness and thresholds. The idea that mathematical reasoning could be in our sense "captured" by a formal system, emerged very late. In particular, the notion of a signature came very late. On the other hand, the axiomatic method has been entrenched in mathematical practice since the time of Euclid. Euclid's axioms were formulated in natural language, and the rules of proof were only implicit; in fact the codification of rules of proof was a long process which took place over centuries, culminating in the work of Frege and his successors. ${ }^{28}$

The question whether the inferential structure of mathematics could be replaced, or modeled, or expressed by ${ }^{29}$ a formalism became urgent around the turn of the twentieth century, as worries about consistency and/or the use of infinitary methods began to impinge on the practice in certain ways and in certain quarters - worries which ripened into, e.g., logicism. The natural question, to what extent mathematics is language entangled, surfaced in an explicit form at the time. Brouwer took a view which prioritized mathematics

\footnotetext{
${ }^{26}$ As Baldwin explains the distinction in his [3]:

It is in this sense that certain recent work of Zilber and Shelah can be seen as developing a formalism-free approach to model theory. Both Zilber's notions of a quasi-minimal excellent class and of a Zariski geometry, and Shelah's concept of an Abstract Elementary Class give axiomatic but mathematical definitions of classes of structures in a vocabulary $\tau$. That is, the axioms are not properties expressed in some formal language based on $\tau$ but are mathematical properties of the class of $\tau$-structures and some relations on it.

${ }^{27}$ Colin McLarty refers to the claim that the actual content of mathematics goes beyond any formalization as "expansive intuitionism," his term for Poincaré's reaction (or counterreaction) to formalism. See his [65]. See also Detlefsen's [17].

${ }^{28}$ Bourbaki calls the distinction between what we have been calling formalism oriented foundations and the axiomatic method, the distinction between logical formalism and the axiomatic method. See [11].

${ }^{29}$ the locution here is very much dependent on one's philosophical perspective.
} 
over language_- "intuitionistic mathematics is an essentially languageless activity of the mind," he said, famously, in [12]; and over formalization in particular, harboring reservations about the importance of the formalization of intuitionistic logic altogether. Fraenkel describes the position this way in his [25]: " ... no formalized theory can do justice to intuitive (which is for them intuitionistic) mathematics or any of its subtheories." And Heyting, who actually axiomatized intuitionistic logic, describes Brouwer's position this way:

... no formal system can be said to represent adequately an intuitionistic theory. There always remains a residue of ambiguity in the representation of the signs, and it can never be proved with mathematical rigour that the system of axioms really embraces every valid method of proof. ${ }^{30}$

Gödel's view of language was similar to Brouwer's in general outline if not in the details, and is best summed up in a remark he once made to Hao Wang: "the overestimation of language," Gödel said, "is deplorable." 31 It lies outside the scope of this paper to treat Gödel's concept of language in any depth. As for formalization altogether, Gödel would hardly have opposed the formalization of "the intuitive." 32 However, the question of faithfulness; the problem arising from the unbridgeable gap between our intuitions and their formal "counterparts," 33,34 so to speak - a problem epitomized by the fact that our axiomatizations often turn out to be non-categorical ${ }^{35}$-was expressed by him in many different forms over his lifetime. ${ }^{36}$ To put it simply, whereas Brouwer saw what one might call the "faithfulness problem" as leading to a wholesale rejection of formal methods, for Gödel the faithfulness problem was rather the explanation for the nonaxiomatizability of mathematics - an essentially critical view of formalization as a totalizing, epistemic project, though one which stops well short of Brouwer's position.

What was the impact of Gödel's Incompleteness Theorems from this point of view? They were the first "threshold results," in bringing to the forefront the relevance of language, in particular of choosing the right vocabulary. The basic picture that emerged is that there is a threshold below which

\footnotetext{
${ }^{30}$ [39], p. 102. Fraenkel comments on the above passage, that in spite of Heyting's "explicit disavowal ... A notion of (intuitionistic) truth can be satisfactorily defined for intuitionistic elementary logic under which the resulting formalized theory is complete and Heyting's logistic system is semantically complete.” See [25], p. 323.

${ }^{31}$ See [85], remark 5.5.7.

${ }^{32}$ For example, Gödel wrote to Wang in 1972 that "Wittgenstein's negative attitude toward symbolic language is a step backward." [85], p. 74.

${ }^{33}$ in David Kazhdan's words, the problem of "the "natural" distinction between the formalism and the substance"

${ }^{34}$ Kazhdan, [46].

${ }^{35}$ i.e., have many non-isomorphic models.

${ }^{36}$ See for example Gödel's letter to Leon Rappaport in [38], pp. 176-177.
} 
one can have desirable properties like decidability, and above which not only decidability is lost but all kinds of pathological behavior occurs. The contemporary model theorist's distinction between "tame" and "wild" structures or theories $[14, \S 4]^{37}$ and the concurrent program of finding important thresholds, pursued by Shelah and his co-workers and culminating in the Main Gap Theorem, are the modern fulfillment of the idea. ${ }^{38}$

Knowing these thresholds, even though they are somewhat subtle combinations of choosing the right vocabulary, the right logic and the right axioms, is now essential for the logician. Our point is this: The role of choosing the right formalism, in either the full or partial sense, rose to prominence but once the thresholds had been recognized, the more formal features that led to the isolation of the thresholds have been abandoned.

3.2. Examples of entanglement. Examples of the entanglement of mathematics with a vocabulary or with a formalism taken in the full sense of the term, abound in logic. In fact, in some sense, mathematical logic can be thought of as the study of just this phenomenon. Here are a few examples of entanglement with a signature:

The real numbers conceived of as the complete ordered field with signature $\langle+, \times,<, 0,1\rangle$, is decidable by Tarski's Theorem. ${ }^{39}$ If one adds to the signature a symbol for the integers, or just the sine function, decidability fails. Similarly Presburger Arithmetic, the structure $\mathbb{N}$ with addition, is decidable, but if $\times$ is added to the vocabulary, it is not.

Zero-one laws for finite structures are sensitive to signature. The probability of a random relational structure on the domain $\{1, \ldots, n\}$ satisfying

\footnotetext{
${ }^{37}$ Though tameness doesn't necessarily imply decidability.

${ }^{38}$ The Main Gap Theorem says, roughly speaking, that given a complete first order theory, either it has - asymptotically - the maximum possible number of non-isomorphic models in all uncountable cardinalities, or it has only "few" models in all uncountable cardinalities. In fact, in the latter, classifiable case, not only is the number of models bounded but one can reconstruct any large model in the class from a relatively small number of invariants. In detail, for countable complete first order theories classifiability (superstable+NDOP+NOTOP+shallow) is a threshold: classifiable theories have relatively few non-isomorphic models in uncountable cardinalities, but unclassifiable theories have the maximal number $2^{\kappa}$ non-isomorphic models in uncountable cardinalities $\kappa$, and the nonisomorphic models can even be chosen so that they are very difficult to distinguish from each other, see [43]. The threshold here is whether a first order theory satisfies those conditions, i.e., superstable, etc., or not. Another threshold involves the characterizability of finiteness.

In another example of a threshold result, a logic, such as $L\left(Q_{0}\right)$ or weak second order logic, in which finiteness can be characterized, can characterize the standard model of arithmetic, and hence the decision problem (the set of Gödel numbers of valid formulas) is non-arithmetical, and the Hanf number is high (at least $\beth_{\omega_{1}^{C K}}$ ). On the other hand, a logic in which finiteness cannot be characterized satisfies a Compactness Theorem which is very close to being effectively axiomatizable, and the Hanf number is (for fully compact logics) just $\omega$. The threshold here is whether the logic is able to characterize finiteness or not. If yes, the logic is "very bad", if not, the logic is "very good"." See Ebbinghaus [21].

${ }^{39}$ I.e., its first order theory is decidable.
} 
a given first order formula tends to either 0 or 1 as $n$ tends to infinity. But if we allow function symbols as part of the language, even the simple sentence $\exists x(f(x)=x)$ has limit probability 1/e. So although one sometimes employs relation symbols and sometimes function symbols, knowing that they are definable from each other, it is not a completely innocuous choice: relational structures satisfy the beautiful zero-one law but once one adds function symbols to these, they do not. ${ }^{40}$

Peano Arithmetic is "logic-entangled" in the following sense: construed as a first order theory, it has continuum many non-isomorphic countable models. However, if it is formalized as a second order theory, it is categorical. Gödel's constructible hierarchy, denoted $L,{ }^{41}$ provides another instance of the entanglement of a canonical structure with a logic. $L$ is built over first order logic. As Myhill and Scott [66] showed, if $L$ is constructed using second order logic, the class obtained is HOD, the hereditarily ordinal definable sets. ${ }^{42}$ In this sense $L$ is sensitive to the underlying logic, at least apparently. Below we will investigate the question to what extent this is a fundamental feature of $L$. Note that $L$, unlike HOD, is absolute in a very strong sense. ${ }^{43}$ In Section 6 of this paper we will discuss extensions of the Myhill-Scott result to other logics.

An instance of entanglement with a formalism in the full sense (so not just with a signature) that has been debated in the literature ever since Quine uttered the pronouncement "Second order logic is set theory in sheep's clothing," [70, page 66], involves the entanglement of the former with the latter. The entanglement of second order logic with ZFC set theory via socalled Large Domain Assumptions, for example, is explained in [81]. ${ }^{44}$ The reader is also referred to C. Parsons's [68]. We return to this point in Section 4.2.2.

These are just a few examples of the widespread phenomenon of entanglement. See also Baldwin's "Completeness and Categoricity (in power):

\footnotetext{
${ }^{40}$ See e.g., [22].

${ }^{41}$ See [44] p. 28 for the definition of the constructible hierarchy.

${ }^{42} \mathrm{~A}$ set $a$ is hereditarily ordinal definable if $a$ itself and also every element of the transitive closure of $a$ is ordinal definable. Both $V=$ HOD and $V \neq$ HOD are consistent, relative to the consistency of ZFC.

${ }^{43}$ Since $L$ is a model of $\mathrm{ZFC}$, the construction of $L$ can be carried out inside $L$. But one obtains by doing this exactly the same structure. This is not true of HOD. For example, if $0^{\sharp}$ exists, it is an element of HOD but not of $L$, thus the HOD of $L$ is different from the actual HOD. In fact, HOD is non-absolute in the even stronger sense that HOD may not satisfy $\mathrm{V}=\mathrm{HOD}$.

${ }^{44}$ In that paper Väänänen suggests that the so-called "second order view" is correct to the extent that mathematics can be done almost entirely within the second order framework. However, the question, where do mathematical structures come from in the first place? persists. If the cumulative hierarchy $V$ is disallowed as a source of structures, one needs large domain assumptions in order to produce sets from which e.g., the reals can be produced, via Comprehension Principles.
} 
Formalization without Foundationalism," [4], a substantive and detailed study of the entanglement of formal logic(s), formal logical syntax, formal systems and the like with "core" mathematics, from the model theoretic perspective. ${ }^{45}$ Our concern in this paper is with the notion dual to entanglement, to which we now turn.

$\S 4$. Examples of formalism freeness. We give some examples of formalism freeness. We begin with examples from mathematical practice and then proceed to some Gödelian projects in, roughly, that direction. We end with some recent developments in model theory.

4.1. The concept of recursive function. This is our main example, as it was the initial point of departure in Gödel's investigation of formalism independence in [31], and it is this: Whether one defines the notion of computability by means of the Gödel-Herbrand-Kleene definition (1936), Church's $\lambda$-definable functions (1936), Gödel-Kleene $\mu$-recursive functions (1936), Turing machines (1936), Post (1943) systems, or Markov (1951) algorithms, ${ }^{46}$ one ends up with the same class of functions. Gödel refers to this phenomenon as formalism independence in his [31]. We return to this example in section 5.1.

4.2. The real numbers. The ordered field of real numbers has great robustness in how its foundations are laid out. Whether one builds the reals as equivalence classes of Cauchy sequences, or as Dedekind cuts, or in some other way, it is remarkable how independent of the set-theoretic definition the reals are. ${ }^{47}$ Robustness also surfaces in a quite different approach to the reals, i.e., the so-called o-minimal structures, a central area of ongoing research in model theory for the last three decades. In the o-minimal structures, as in the reals, the (first order) definable sets are just finite unions of (half)open intervals, and their complements. Real closed fields, of which the field of reals is an example, are all o-minimal, as is the field of real numbers with exponentiation as an added function. ${ }^{48}$

\footnotetext{
${ }^{45}[4]$ is a companion, so to speak, to his "Formalization, Primitive Concepts and Purity" [3] in the sense that the while the latter is a treatment formalism freeness (and other issues), the former is a treatment of entanglement.

${ }^{46}$ See, e.g., [15].

${ }^{47}$ This robustness of the real numbers underlies a phenomenon John Burgess calls "indifferentism," in a recent paper, [13], namely the "general phenomenon of the indifference of working mathematicians to certain kinds of decisions that have to be made in any codification of mathematics ... two analysts who wish to collaborate do not need to check whether they were taught the same definition of "real number"." Burgess traces the advent of indifferentism to the mid-19th century, when mathematicians established the arithmetization of analysis, freeing it from its geometrical foundations.

${ }^{48}$ By Wilkie's theorem. Of course, the robustness of the concept of o-minimality has to do not with independence of the set-theoretic definition, but rather with yielding consequences for many theories that are apparently only distantly related.
} 
4.2.1. The concept of an ordered pair. The set-theoretical concept of an ordered pair is often cited as manifesting (in set theory) an artificial dependence on notation, namely Kuratowski's notation $\langle a, b\rangle=\{\{a\},\{a, b\}\}$. That the accusation is based on a misunderstanding - the set theorist's dependence on the notation is not a genuine one-does not make the example less illuminating. What is at issue is the idea that concepts in set theory, such as the ordered pair concept, are defined uniquely and not up to isomorphism. However this does not indicate an attachment to any particular way of presenting the unique object in question. Set theory is invariant under the choice of how things like ordered pairs are actually defined. In the end, the set theorist's reliance on the formalism $\langle a, b\rangle=\{\{a\},\{a, b\}\}$ extends only as far as the recognition that the ordered pair can be so defined. But then the formalism is ignored, having served its purpose. ${ }^{49}$

4.2.2. What is the logical foundation of mathematics? We consider the other extreme (from the concept of ordered pair), namely mathematics itself, i.e., mathematics taken as a whole. The question the formalism oriented foundationalist is concerned with is the following: is there a single formalism which can serve as the language in which all of mathematics can be expressed? Some have argued that ZFC set theory is one such formalism, while an earlier alternative is higher order logic. Since higher order logic and first order set theory have very different "metalogical" properties, one may ask, is one superior to the other-if one can really choose between them at all. Väänänen has argued that mathematics altogether is indifferent to such a choice of logic, especially when that choice is between first order set theory and higher order logic. From the practical point of view, the working mathematician will — and should - be indifferent to the choice between these two theories, and there are deep theoretical reasons why this should be the case:

We study two metatheories of mathematics: first-order set theory and second-order logic. It is often said, that second-order logic is better than first-order set theory because it can in its full semantics axiomatize categorically $\mathbb{N}$ and $\mathbb{R}$, while first-order axiomatization of set theory admits non-standard, e.g., countable models. We show below that this difference is illusory. If second-order logic is construed as our primitive logic, one cannot say whether it has full semantics or Henkin semantics, nor can we meaningfully say whether it axiomatizes categorically $\mathbb{N}$ and $\mathbb{R}$. So there is no difference between the two logics: first-order set theory is merely the result of extending second-order logic to transfinitely high types. ${ }^{50}$

\footnotetext{
${ }^{49}$ The exact form of the ordered pair comes up only in some rather trivial rankcomputations in set theory.

${ }^{50}$ See [80], p. 506-507.
} 


\subsection{Some Gödelian projects.}

4.3.1. Constructibility; Turing machines. We argued above that Gödel's constructible hierarchy $L$ is entangled with first order logic, in virtue of the fact that if the underlying first order logic is changed to second order logic a (seemingly) entirely different structure emerges, namely HOD. However, we will argue that $L$, in its first order version, also manifests a high degree of formalism freeness. This is discussed at length in Section 6, but we make already here the following observation: Gödel's original presentation of $L$ was given in terms of first order definability in set theory. ${ }^{51}$ In a second monograph, ${ }^{52}$ Gödel presented $L$ in terms of the so-called Gödel (or rudimentary) functions. The latter presentation is formalism free in the sense that there is no logical formalism present, rendering constructibility completely intelligible to the non-set theorist.

A similar phenomenon emerges with the notion of recursive function, a notion which admits many different formalizations, as was noted above. The presentation in terms of Turing machines ${ }^{53}$ resembles, in its mathematical naturality, the representation of constructibility in terms of Gödel functions, in that again no explicit formalism is present. ${ }^{54,55}$ We return to computability in section 5.1.

4.3.2. Dialectica. The system $T$ of Gödel's so-called "Dialectica Interpretation" 56 interprets Heyting's intuitionistic arithmetic in terms of so-called computable functionals of finite type. This gives a consistency proof for Heyting arithmetic and thus for Peano Arithmetic, if one combines the Dialectica Interpretation with Gödel's earlier double-negation interpretation of Peano Arithmetic into Heyting Arithmetic.

\footnotetext{
${ }^{51}$ See equations (1) in Section 6.

${ }^{52}[30]$.

${ }^{53} \mathrm{~A}$ Turing machine is ultimately just a finite set of 4-tuples of natural numbers.

${ }^{54}$ The link between these two concepts is further emphasized by the fact that infinitary Turing machines actually generate $L$, [52].

${ }^{55}$ Gödel credited Turing with giving a fully general, mathematical characterization of the concept of a "formal system," remarking that

This concept is shown to be equivalent with that of a "Turing machine." A formal system can simply be defined to be any mechanical procedure for producing formulas, called provable formulas.
}

This is a postscript added to the 1965 reprinting of his Princeton lectures in the volume [16], reproduced in [34, p. 369].

Gödel's identification of a formal system as a mechanical procedure for producing provable formulas, would restrict the notion of a formal system to those with at most a recursively computable provability predicate. This rules out many of the purely semantically defined formalisms such as (full) second order logic with the semantic consequence relation. (A sentence $\phi$ is a semantic consequence of a sentence $\psi$ if every model of $\psi$ is a model of $\phi$.

${ }^{56}[33]$. 
In his introduction to [33], ${ }^{57}$ Troelstra notes the logic-freeness of Gödel's system $T$ :

... Gödel did not want to go as far as admitting Heyting's abstract notion of constructive proof; hence he tried to replace the notion of constructive proof by a more definite, less abstract (that is, more nearly finitistic) notion, his principle candidate being a notion of "computable function of finite type" which is to be accepted as sufficiently well understood to justify the axioms and rules of his system $T$, an essentially logic-free theory of functionals of finite type. ${ }^{58}$

In a crucial passage of the paper Gödel explains the move to replace the notion of a Heyting proof with a more concrete, perspicuous, but still abstract notion. The choice is forced by the Second Incompleteness Theorem, which demonstrates that the consistency proof will require abstract notions going beyond what Gödel calls the finitary attitude:

Here by abstract (or nonintuitive) we must understand those that are essentially of second or higher order, that is, notions which do not involve properties or relations of concrete objects, (for example, combinations of signs), but that relate to mental constructs (for example, proofs, meaningful statements and so on); and in the proofs we make use of insights, into these mental constructs, that spring not from the combinatorial (spatiotemporal) properties of the sign combinations representing the proofs, but only from their meaning. ${ }^{59}$

In the paper Gödel takes as a primitive the notion of intuitionistic evidence, rather than the concept of a Heyting proof. Relevant to our concerns here is Gödel's suggestion, that for a consistency proof it is not enough to work with "(spatiotemporal) properties of the sign combinations representing the proofs"; one must work with the concept of meaning directly. ${ }^{60}$ Gödel struggled to give a logic-free construction of it, analogous, perhaps, to that which he provided for the notion of constructibility in set theory.

Another example of formalism freeness in the setting of constructive mathematics is Bishop's Foundations of Constructive Analysis [10], in which Bishop gives a relatively formalism free presentation of mathematical analysis in a constructive framework.

\footnotetext{
57 "Note to 1958 and 1972 " in [35].

${ }^{58}$ [35], p. 221. Italics ours.

${ }^{59}$ See Gödel's [1958], in [35], p. 241.

${ }^{60}$ The presentation of the system $T$ is obscure in places, and as an attempt at formalism freeness, it fails. In Gödel's late correspondence with Bernays, Bernays questioned whether the notion of constructive proof is not needed after all-is it not implicit in the concept of computable function of finite type? See [37].
} 
4.3.3. Gödel's notion of "solvability by all means imaginable". We now consider some remarks from Gödel's 1929 thesis on the notion of "absolute provability," which is really a formalism free concept of provability. ${ }^{61}$ Gödel draws the distinction there between formal provability and what Gödel will later call absolute provability, or provability "through all specified means." More precisely, he observes that one might raise the following objection to the main result of his thesis: doesn't the use of the law of excluded middle in its proof "invalidate the entire completeness proof"? The Completeness Theorem asserts

'a kind of decidability,' namely every quantificational formula is either provable or a counterexample to it can be given, whereas the principle of the excluded middle seems to express nothing other than the decidability of every problem. ${ }^{62}$

Thus the proof may be circular: one assumes the decidability of every question in order to prove just that assertion. ${ }^{63}$

But, Gödel goes on to say, what he has shown is the provability of a valid formula from "completely specified, concretely enumerated inference rules," 64 not merely from all rules imaginable; whereas the law of excluded middle is used informally in the sense that the notion of decidability or solvability asserted by the law is left unspecified. As Gödel puts it:

... what is affirmed (by the law of excluded middle) is the solvability not at all through specified means but only through all means that are in any way imaginable ... ${ }^{65}$

The Completeness Theorem, then, provides a reduction: if we assume solvability by all means imaginable, then we have, in the case of a sentence of first-order predicate calculus, a reduction to solvability by very specific means laid out beforehand. ${ }^{66}$

In fact Gödel took the view in the 1930s and early 1940s, that certain other mathematical problems may very well be absolutely undecidable, ${ }^{67}$ a view he

\footnotetext{
${ }^{61}$ Some of this material on Gödel's 1929 thesis is adapted from the author's [49].

${ }^{62}[34]$, p. 63.

${ }^{63}$ This objection to the Law of Excluded Middle is the content of Brouwer's "Third Insight" as expressed in his "Intuitionistische Betrachtungen über den Formalismus." See p. 40, [63] for an English translation.

${ }^{64}$ italics Gödel's.

${ }^{65}$ ibid.

${ }^{66} \mathrm{Gödel}$ remarks in a footnote to this passage that the notion of provability "by any means imaginable" is perhaps "too sweeping" - a hesitation to which he is no longer subject by the mid-1940s. See [34], p. 65.

${ }^{67}$ In 1939, for example, Gödel explained his consistency proofs of $\mathrm{AC}$ and $\mathrm{CH}$ in a lecture in Göttingen and on that occasion he voiced his suspicion that the axiom of constructibility, $V=L$, would be strongly absolutely undecidable:
}

The consistency of the proposition $A$ (that every set is constructible $[V=L]$ ) is also of interest in its own right, especially because it is very plausible that with $A$ 
will later discard. ${ }^{68}$ The incompleteness theorems do not, in the end, destroy the Hilbert program "in its original extent and meaning":

As to problems with the answer Yes or No, the conviction that they are always decidable remains untouched by these results. ${ }^{69}$

Gödel's goal subsequent to this change of mind was the establishment of an informal decision procedure - an idea logicians would hardly countenance today. Kreisel entertained the idea in some form in 1972, ${ }^{70}$ though he fell short of actually recommending the development of such a procedure.

According to Tarski such a procedure could not be given, as his correspondence with Gödel just prior to the 1946 Princeton Bicentennial meeting ${ }^{71}$ reveals:

I am so glad that you are planning to talk at the Princeton Conference about the notions of absolute provability, definability, etc.... As regards the question in which you are interested, I don't think I can do anything else but to emphasize the fundamental difference between all the undecidable statements known at present in elementary number theory on the one hand and some undecidable statements (like [the] continuum hypothesis) in analysis and set theory; the statements of the first kind being clearly undecidable in a relative sense while those of the second seem to be undecidable

\footnotetext{
one is dealing with an absolutely undecidable proposition, on which set theory bifurcates into two different systems, similar to Euclidean and non-Euclidean geometry. $([* 1939 b]$, in [36], p. 155)
}

${ }^{68}$ Gödel was likely aware of Luzin's remark in this vein in 1925, but for the projective sets: "One does not know and one will never know of the family of projective sets, although it has cardinality $2^{\aleph_{0}}$ and consists of effective sets, whether every member has cardinality $2^{\aleph_{0}}$ if uncountable, has the Baire property, or is even Lebesgue measurable," see [58].

${ }^{69}$ [193?] in [35]. Gödel would later expand on these remarks in a discussion about Leibniz's characteristica universalis with Carnap in 1948:

The universal characteristic claimed by Leibniz (1677) does not exist. Any systematic procedure for solving problems of all kinds would have to be nonmechanical. (Gödel Nachlass, folder 1/209, 013184, p. 1.)

See [82] for the concept of strong absolute undecidability as well as for an extensive discussion of Gödel's views on absolute undecidability during the period of the 1930s and early 40s.

${ }^{70}$ Kreisel [54], p. 322:

[I]t has been clear since Gödel's discovery of the incompleteness of formal systems that we could not have mathematical evidence for the adequacy of any formal system; but this does not refute the possibility that some quite specific system ... encompasses all possibilities of (correct) mathematical reasoning ... In fact the possibility is to be considered that we have some kind of nonmathematical evidence for the adequacy of such [a system].

\footnotetext{
${ }^{71}$ at which he and Gödel were both scheduled to speak at a session Tarski had organized
} on undecidability. 
in some absolute sense. And in this connection I shall raise the problems (1) whether [and how] the notions of relative and absolute undecidability can be made precise and (2) whether, on the basis of some adequate definition of these notions, it will be possible to show that a number-theoretic problem can be undecidable only in a relative sense ... ${ }^{72}$

Gödel's views on absolute definability came to fruition in his remarks to the 1946 Princeton Bicentennial Conference, in which he suggests - as we interpret that lecture-developing a form of the Church-Turing thesis for the notion of definability in set theory. ${ }^{73}$

We now take up certain developments in model theory, an inspiration behind the ideas in this paper, along with Gödel's 1946 Lecture.

4.4. Model theory. Model theory is that part of mathematical logic which arose from the realization that Gödel's Completeness Theorem gives a very rich plethora of models of first order theories. Despite the origins of model theory being firmly embedded in the development of formal systems and their models, a number of modern developments seem to undermine this.

4.4.1. Abstract elementary classes. In the 1980s Saharon Shelah, when trying to develop the model theory of infinitary languages ${ }^{74}$ and their extensions by generalized quantifiers, eventually decided to dispense (in this context) with the language altogether ${ }^{75}$ and merely state the properties he needed classes of models to satisfy, whether these properties arose from the syntax and semantics of any particular infinitary language or not. Model classes that satisfy these assumptions came to be known as AECs (abstract elementary classes). A typical non-trivial assumption is that the model class is closed under unions of chains with respect to an abstract (strong) submodel relation, which mimics the elementary submodel relation. Shelah in a sense isolated from infinitary languages the part that was susceptible to model-theoretic development.

Subsequently the connection to infinitary languages has been forgotten and AECs are studied on their own. ${ }^{76}$ A typical test question is to prove some form of Morley's Theorem. We can say that to isolate the axioms of AECs (such as the closure under unions of chains) and to recognize their importance, it was essential to have the language in the background, but once this initial stage was over, the language could be dispensed with.

\footnotetext{
${ }^{72}$ Notably, the undecidability of the $\mathrm{CH}$ was not established until 1963.

${ }^{73}$ For more on Gödel's notion of absolute provability, see [29], reprinted in [34].

${ }^{74}$ We use "language" here as meaning a logic, not a vocabulary.

${ }^{75}$ The trend toward language freeness in contemporary model theory was a primary influence on the development of the ideas of this paper.

${ }^{76}$ See however [55], which represents an interesting "return" to the roots of AECs in infinitary languages.
} 
In the abstract elementary class framework there is a sense in which a syntactic concept such as the concept of a formula (or more exactly of a "type") becomes, simply, a set invariant under automorphisms ${ }^{77}$ — though strictly speaking, in the AEC framework there simply is no notion of formula. This is just one example of the way a syntactic concept is absorbed by a semantic notion in the AEC framework.

4.4.2. Stability and other model-theoretic themes. In certain stability theoretic contexts there is likewise a tendency to emphasize, over the underlying first order logic, the relevant semantic characterization of the logical concept. (Although properties of first order logic, such as the Compactness Theorem, will always emerge in other ways.) What is important for the geometric or algebraic study of structures in stability theory are the closure and independence properties of definable sets and types, among other central concepts, ${ }^{78}$ and they can, arguably, be simply listed without any reference to the syntax and semantics of first order logic. ${ }^{79}$ What is relevant here are facts of the following kind: that quantifier elimination holds in a (one-dimensional) Zariski structure, for example, is just the statement that the projection of a constructible set is constructible.

This development has transformed the model theorist's study of the "old" concrete objects, such as algebraic varieties and analytic curves, into one in which these are viewed in a new framework, one which generalizes the classical approaches, but is spelled out with no reference to formal languages or their properties.

For a finely grained and penetrating analysis of various formalism free phenomena in the vast terrain that is contemporary model theory, and in particular in the AEC and stability theoretic context, the reader is referred to John Baldwin's "Formalization, Primitive Concepts and Purity." example, about Shelah's presentation theorem ${ }^{81}$ Baldwin remarks:

\footnotetext{
${ }^{77}$ Assuming the so-called Amalgamation Property, and arbitrarily large models in the class. This is the notion of a Galois type over a model, essentially an orbit under a group action- "an entirely mathematical concept." Baldwin, personal correspondence.

${ }^{78}$ As A. Villaveces puts it, "[also important] are various geometrical configurations, genericity of types, eventual behavior of types (stationarity), domination of types, orthogonality of types ... "the new primitives". In FO Stability, of course, all their definitions are given syntactically, at least initially. However, many of these benefit from general, non-logical, formalism free, semantic characterizations. For instance, Adler [1] ... characterizes forking and thorn-forking purely in terms of a "geometry of possible extensions" of models and types (orbital types), ultimately giving up formulas (where the original definitions were apparently very formula-laden)." (A. Villaveces, personal communication.)

${ }^{79}$ E.g., the Zariski structures of Zilber and Hrushovski [42], or the Tarski systems of van den Dries [83].

${ }^{80}[3]$.

${ }^{81}$ Shelah's presentation theorem asserts that an AEC with arbitrarily large models can be defined as the reducts of models of a first order theory which omit a family of types.
} 
"passing through the syntax, Shelah obtains a purely semantic theorem ... The syntactic condition in the theorem is a set of sentences in roughly Tarski's sense ... but we are able to deduce purely semantical conclusions."

For Baldwin the key concept here is that of an implicit syntax, and in particular the essential use of formalism to obtain a theorem which is stated in a formalism free manner. ${ }^{82}$ About Zilber's notion of quasi-minimal excellent class, Baldwin remarks:

In contrast, Zilber's notion of a quasi-minimal excellent class [88] was developed to provide a smooth framework for proving the categoricity in all uncountable powers of Zilber's pseudo-exponential field ... The key point is that there are no axioms in the object language of the general quasiminimal excellence theorem; there are only statements about the combinatorial geometry determined by what are in the application the $L_{\omega_{1} \omega}$-definable sets.

As Baldwin points out, whereas the presentation theorem passes through syntax to get a formalism free statement, in a kind of converse, Zilber's result "passes through a (substantial) formalism free step in the argument to get a formal result - a categorical theory axiomatized in a formal language." 83

This is a very cursory look at a vast subject. The reader is also referred to Hodges's "What is a Structure Theory?", in particular the discussion around the remark that "The difficulties about aligning algebra with logic haven't prevented Shelah from using the notion of superstability to prove results with a clear algebraic content." 84

We mentioned the role of Gödel's Completeness Theorem in the development of what one might call pure semantic methods in model theory. But this approach also has its roots in the semantic point of view developed by Tarski and others: ${ }^{85}$

4.4.3. Tarski's notion of "mathematical property". In his [3] Baldwin notes the distinction between a mathematical as opposed to logical property. As examples he cites the following:

- (Tarski) A class of structures in a finite relational language is universally axiomatizable if and only if it is closed under isomorphism, substructure and if for every finite substructure $B$ of a structure $A, B \in K$ then $A \in K$.

- (Birkhoff) A class K of algebras is axiomatized by a set of equations if and only it it is closed under homomorphisms, subalgebras, and direct products.

\footnotetext{
${ }^{82}$ Baldwin, personal communication and [3].

${ }^{83}$ Baldwin, op cit.

${ }^{84}$ p. 214, [41]. Italics ours.

${ }^{85}$ Notably the algebraic tradition in logic associated with Peirce and Schröder.
} 
Baldwin remarks about the notion of a mathematical as opposed to a logical property, that:

An inquiry can be 'formalism-free' while being very careful about the vocabulary but eschewing a choice of logic and in particular any notion of formal proof. Thus it studies mathematical properties in the sense we quoted from Tarski above.

It is in this sense that certain recent work of Zilber and Shelah can be seen as developing a formalism-free approach to model theory. Both Zilber's notions of a quasi-minimal excellent class [59] and of a Zariski geometry [29], and Shelah's concept of an Abstract Elementary Class [49] give axiomatic but mathematical definitions of classes of structures in a vocabulary $\tau$. That is, the axioms are not properties expressed in some formal language based on $\tau$ but are mathematical properties of the class of structures and some relations on it. ${ }^{86}$

Much of the model theory in the 1950s involved the search for mathematical descriptions of canonical model classes. In the same vein, also in this period, Scott [73] investigated the possibility of finding so-called natural non-standard models of Peano Arithmetic. These are constructed from recursive functions rather than by recourse to the Completeness Theorem. A very similar approach building on extensions of Skolem's construction of a non-standard model of Peano Arithmetic was undertaken in [48].

4.4.4. First order logic. According to so-called Lindström's Theorem ${ }^{87}$ first order logic is the maximal logic which satisfies two basic model theoretic properties, the Downward Löwenheim-Skolem Theorem and the Compactness Theorem. This means that one can view first order logic purely semantically with no concern as to the syntax. As long as these two model theoretic properties are satisfied, the concept of a definable model class is the same. As far as definability of model classes is concerned, first order characterizability manifests then, thanks to Lindström's Theorem, very strong formalism freeness.

In fact, many other logics permit a semantic characterization. Barwise ${ }^{88}$ showed that if $\kappa=\beth_{\kappa}$, then $\mathcal{L}_{\kappa \omega}$ is the maximal logic which has the Karp Property ${ }^{89}$ and well-ordering number ${ }^{90}$ is at most $\kappa \cdot{ }^{91}$ This is not as sharp a semantic characterization as Lindström's, although Barwise relies heavily on Lindström's proof; but the two conditions are in principle comparable

\footnotetext{
${ }^{86}[3]$.

${ }^{87}[56]$.

${ }^{88}$ See [8].

${ }^{89}$ partially isomorphic structures are elementarily equivalent with respect to the logic.

${ }^{90}$ the smallest ordinal $\alpha$ such that if a sentence of the logic has only models in which a binary predicate $R$ is well-ordered, then in every model the order type of $R$ is $<\alpha$.

${ }^{91}$ [8].
} 
to the conditions occurring in Lindström's proof, namely the LöwenheimSkolem and Compactness Theorems. Thus $\mathcal{L}_{\kappa \omega}$, for $\kappa=\beth_{\kappa}$, manifests some extent of formalism freeness, in the sense that its class of definable model classes is not entirely entangled with the ordinary syntax of $\mathcal{L}_{\kappa \omega}$. Another interesting case is the recent logic $L_{\kappa}^{1}$ of Shelah [77], which is between $\mathcal{L}_{\kappa \omega}$ and $\mathcal{L}_{\kappa \kappa}$ and satisfies a Lindström Theorem. What is interesting is that Shelah does not actually give a syntax for this logic; the syntax is not "needed" because there is already a semantic characterization, via reference to its Ehrenfeucht-Fraïssé game. ${ }^{92}$ Yet another class of infinitary languages which demonstrates remarkable formalism freeness, also due to Barwise [7], is the family of admissible fragments $\mathcal{L}_{A}$ of $\mathcal{L}_{\infty \omega \omega}$. If $A$ is an admissible set, then the language $L_{A}$ is the maximal strictly absolute ${ }^{93}$ logic defined with parameters in $A$. So the syntax of $\mathcal{L}_{A}$ does not "matter" as long as it is strictly absolute. ${ }^{94}$

4.4.5. Karp on "varying the logic". Carol Karp made extensive use of the infinitary languages $\mathcal{L}_{\kappa, \lambda}$ in her study of properties of Boolean Algebras. In an unfulfilled research project ${ }^{95}$ she suggested that certain recursiontheoretic applications could be carried out while circumventing coding. Lopez-Escobar [57] describes Karp's research project as follows:

Although Carol Karp appreciated recursive function theory, she disliked proofs which involved codings and systems of notations. In her work on infinitary set theory she noticed that infinitely long formulae sometimes allowed her to circumvent notations. She then tried treating the recursive set functions as functions representable in infinitary systems using implicit definitions. She discovered that by varying the $\operatorname{logic}^{96}$ in the system one could get a host of results about recursion theory and its extensions; furthermore it could be done without any ad hoc notations.

It was her research on the infinitely long formal proofs that led Karp to the concept of $L$-R.E. ${ }^{97}$ on A. However, it is clear that the actual structure of the proofs is irrelevant, for all that is ever used is the consequence relation. Thus, for the purpose of discussing extensions of recursion theory, it does not make much sense to dwell too much upon the axioms and rules of inference. Consistency properties are a natural way of getting all the benefits of completeness while, at the same time, avoiding formal proofs.

\footnotetext{
${ }^{92}$ The game is like the usual Ehrenfeucht-Fraïssé game of $L_{\kappa \kappa}$ (see e.g., Dickmann [20]), but the second player can delay her responses in a way which positions the logic between $L_{\kappa \omega}$ and $L_{\kappa \kappa}$.

${ }^{93}$ I.e., sentencehood is $\Sigma_{1}^{K P}$ and the satisfaction predicate is $\Delta_{1}^{K P}$.

${ }^{94}$ We thank Jouko Väänänen for the examples in this paragraph.

${ }^{95}$ unfulfilled due to her early death.

${ }^{96}$ italics ours.

${ }^{97} L$-recursively enumerable.
} 
Below we also consider "varying the logic in the system," in the context of definability in set theory. This is our main example of the notion of formalism freeness.

4.5. Summarizing the examples. Formalism freeness, a phenomenon which, it is hoped, the reader now sees as a genuine one, even in this brief outline, is ubiquitous. A classification of these different modes of formalism freeness into (likely overlapping) categories, might run along the following lines: (1) "Logic independence," as evidenced in the Karp project, and also in ours below in Section 6 with respect to definability. (2) Admitting a mathematical rather than logical treatment, as exemplified by constructibility given in terms of Gödel functions; Turing machines; AEC's; certain aspects of stability theory; the notion of computable function of finite type; presentations of the reals. (3) Transcendence (even local transcendence) with respect to a logical hierarchy. ${ }^{98}$ (4) Formalism independence in the sense of stability under a class of presentations, viz computability.

One can think of theses various modes of the concept of formalism freeness as the simple preference for semantic methods, i.e., methods which do not involve or require the specification of a logic - at least not prima facie. Formalism freeness is manifest in the distinction between the socalled axiomatic method and the method of formal systems, as we have noted. For example, Euclidean geometry was given initially as an axiomatic system, but it was formalized - in a preliminary sense - in Hilbert's 1899 Grundlagen der Geometrie. ${ }^{99}$ Formalism freeness is in some sense an aspiration - unfulfillable perhaps, given what appears to be at least an apparent entanglement of mathematics with the various set- or category-theoretic formalisms - formalisms which do succeed, after all, in codifying almost the entire inferential structure of mathematics. Formalism freeness involves a leap of faith, involving as it does a move away from the support of formal methods. ${ }^{100}$ This is, in some sense, the main dilemma here - the aesthetic question, if you will. One seeks a natural, unentangled mathematical concept.

§5. Gödel's remarks to the 1946 Princeton Bicentennial Conference. We now take up our main point of departure for the mathematical ideas in this paper, Gödel's [31]. Before considering the address in detail, a brief summary. Gödel begins by grouping together three epistemological notions, as he calls them: computability, definability and provability. Note that each of these come with their own paradoxes, which, with some care, can be turned into theorems. ${ }^{101}$ With computability we have the unsolvability

\footnotetext{
${ }^{98}$ See below and see Gödel's [31].

${ }^{99}[40]$.

${ }^{100}$ at least, insofar as those methods involve formalization.

${ }^{101}$ Gödel mentions paradoxes explicitly only in connection with definability.
} 
of the halting problem, self-reproducing Turing machines, and so on; with provability there are the Incompleteness Theorems; with the concept of definability paradoxes arise from the attempt to define definability. For example, "the least undefinable ordinal" defines an ordinal, paradoxically. ${ }^{102}$

It is not clear what Gödel means by the word "epistemological," beyond the fact that all of these involve the activity of the mathematician. The goal here is to see that each of computability, definability and provability admits an "absolute" version, and indeed what is explained in the Princeton address is how to formulate "absolute" or in a special sense formalism independent, and at the same time non-paradoxical versions of the two epistemological notions of provability and definability. ${ }^{103}$ That this is at all possible is testimony to the great robustness manifested by these concepts.

As to the address itself, there are no proofs in it, only remarks and conjectures. Strikingly, in most cases the methods involved in the proofs, had they been given, were not developed until much later. For example, Gödel indicates that ordinal definability is itself definable, a fact which is nowadays proved using the Levy Reflection Principle. ${ }^{104}$ Gödel also asserts here (not conjectures) the independence of the axiom of constructibility, $V=L{ }^{105}$ Another conjecture of Gödel's is that HOD ${ }^{106}$ will be a model of set theory satisfying choice, giving a simpler consistency proof of the Axiom of Choice, which was proved in [66]. Finally, Gödel predicts that the proof of AC in $\mathrm{HOD}$ will not extend to a proof of the $\mathrm{CH}$, and in fact the failure of the $\mathrm{CH}$ was shown to be consistent with $V=$ HOD in $1968 .{ }^{107}$

5.1. Computability. We now consider the lecture in detail. Gödel begins by addressing the concept of computation. He points out that this concept can be given a formalism independent definition:

Tarski has stressed in his lecture the great importance (and I think justly) of the concept of general recursiveness (or Turing computability). It seems to me that this importance is largely due

\footnotetext{
${ }^{102}$ Other definability paradoxes include the Berry paradox, "the smallest integer not definable by at most ten words," the Richard paradox, and so on.

${ }^{103}$ Gödel's notion of formalism independence for definability and provability involves a conception which embraces all the steps in a transfinite hierarchy at once. See below.

${ }^{104}$ The principle says that for every $n$ there are arbitrarily large ordinals $\alpha$ such that $V_{\alpha} \prec_{n} V$.

${ }^{105}$ In this connection see Gödel's letter to Wolfgang Rautenberg of June 30, 1967, in [38], p. 183.

${ }^{106} \mathrm{HOD}$ is the class of hereditarily ordinal definable sets. OD is the class of ordinal definable sets, i.e., those sets which are definable by a formula of set theory with finitely many ordinal parameters. In the address, Gödel only mentions OD; but we take him to be referring to HOD in most cases.

${ }^{107}$ See [64]. This raises the question whether HOD was Gödel's candidate for the consistency of the failure of the $\mathrm{CH}$.
} 
to the fact that with this concept one has succeeded in giving an absolute definition of an interesting epistemological notion, i.e., one not depending on the formalism chosen. ${ }^{108}$

Gödel could be referring to the fact that whether one defines the notion of computability by means of the Gödel-Herbrand-Kleene definition (1936), Church's $\lambda$-definable functions (1936), Gödel-Kleene $\mu$-recursive functions (1936), Turing machines (1936), Post (1943) systems, or Markov (1951) algorithms, ${ }^{109}$ one defines the same class of functions (as was noted above). Gödel used the phrase "formalism independent" for this mode of formalism freeness. But another sense of formalism independence emerges in the paper, having to do with "the absence of the sort of relativity to a given language that leads to stratification of the notion such as (in the case of definability in a formalized language) into definability in languages of greater and greater expressive power." 110 Restricted to computability, this is the idea that one cannot diagonalize out of the class of partial recursive (or computable) functions, in the way one can diagonalize out of the class of total recursive functions.

In a footnote Gödel added in $1965,{ }^{111}$ he clarifies the notion of formalism independence, a broadened absoluteness claim ${ }^{112}$ at stake in this passage:

To be more precise, a function of integers is computable in any formal system containing arithmetic if and only if it is computable in arithmetic, where a function $f$ is called computable in $S$ if there is in $S$ a computable term representing $f .{ }^{113}$

The concept of partial recursive function is then in some sense saturated for arithmetic; that is to say, there is no computable (i.e., partial recursive) function which is computable in a system extending arithmetic, which is not already computable in arithmetic. Gödel contrasts this situation with the apparently less felicitous cases of provability and definability, remarking that:

In all other cases treated previously, such as demonstrability of definability, one has been able only to define them relative to a given language, and for each individual language it is clear that the one thus obtained is not the one looked for. For the concept of computability, however, although it is merely a special kind of demonstrability or definability, the situation is different. By a

\footnotetext{
${ }^{108}$ [35], p. 150 .

${ }^{109}$ See, e.g., [15].

${ }^{110}$ Parsons, introductory note to [1946].

${ }^{111}$ To the version published in [16].

112 according to Sieg, see [78].

${ }^{113}$ italics ours.
} 
kind of miracle it is not necessary to distinguish orders, and the diagonal procedure does not lead outside the defined notion. ${ }^{114}$

There is an important unstated context here, involving Turing's analysis of the notion of "formal system" as it bears on the question of the generality of the First Incompleteness Theorem, and in turn with the establishment of the absoluteness of the concept of computability. The absoluteness was needed for settling the question, taken up by Gödel himself in his 1931 paper on the Incompleteness Theorems, whether those theorems are completely general, that is, whether they apply to any formal system containing arithmetic, and not just Principia and systems related to it. Gödel was careful to say at the end of his 1931 paper that this had not been shown. But Turing's analysis lays this doubt to rest for Gödel. ${ }^{115}$ In a postscript Gödel added on the occasion of the reprinting of his Princeton lecture in Martin Davis's 1965 The Undecidable, he writes:

In consequence of later advances, in particular of the fact that, due to A. M. Turing's work, a precise and unquestionably adequate definition of the general concept of formal system can now be given, the existence of undecidable arithmetical propositions and the non-demonstrability of the consistency of a system in the same system can now be proved rigorously for every consistent formal system containing a certain amount of finitary number theory. ${ }^{116}$

The generality issue ${ }^{117}$ arises in the first place because the general notion of formal system is not given in the 1931 paper. But, Gödel continues:

Turing's work gives an analysis of the concept of "mechanical procedure" (alias algorithm or computation procedure or "finite combinatorial procedure"). This concept is shown to be equivalent with that of a "Turing machine." A formal system can simply be defined to be any mechanical procedure for producing formulas, called provable formulas. For any formal system in this sense there exists one in the [usual] sense that has the same provable formulas (and likewise vice versa) ... 118,119

\footnotetext{
${ }^{114}$ As we remarked above about the notion of computability given in terms of Turing machines particularly, not only "is [it] not necessary to distinguish orders, and the diagonal procedure does not lead outside the defined notion," but the concept is apparently logic free relative to first order logic.

${ }^{115}$ Kreisel takes a similar line in his [53].

${ }^{116}[34]$, p. 369.

${ }^{117}$ As we noted above, there is an interesting literature on the question of the generality of the Second Incompleteness Theorem, having to do with concerns about the intensional correctness of the relevant consistency statements, also in weak arithmetic theories. See references in footnote 7 .

${ }^{118}$ ibid.

${ }^{119}$ As we noted above, Gödel's identification of a formal system as a mechanical procedure for producing provable formulas, would restrict the notion of a formal system to those
} 
If the meaning of Turing's analysis (in Gödel's mind) was initially bound up with the generality issue, for Gödel in subsequent years the meaning of the Turing analysis, in particular the suggestion that mechanization and formalization are in some sense equivalent, was that it both precipitated and crystallized the distinction between the mechanizable activity of formalization, and the activity of the mathematician proper. As Gödel would later write to Leon Rappaport:

My theorems only show that the mechanization of mathematics, i.e., the elimination of the mind and of abstract entities, is impossible, if one wants to have a satisfactory foundation and system of mathematics. ${ }^{120}$

To Gödel in 1946, however, what was important about the Turing analysis was not its solution of the generality issue per se, or the fact that it pushes the abstract entities of mathematics into "human territory," so to speak, as the remark to Rappaport might suggest; rather (or in addition) what was important about the Turing analysis to Gödel at the time, was that it represented a methodological ideal.

For Gödel (and others), Turing answered the question: what can a person compute? definitively and finally, by laying out a set of mechanical operations which, in the right combinations and carried out in the right way, reduce the computation of, e.g., a $\lambda$-definable function, to something very much like child's play. ${ }^{121}$ This is achieved by exhibiting a (in some sense) perfect model of human calculability; as Gödel would later put to Hao Wang

The resulting definition of the concept of mechanical by the sharp concept of "performable by a Turing machine" is both correct and unique ... Moreover it is absolutely impossible that anybody who understands the question and knows Turing's definition should decide for a different concept. ${ }^{122}$

Wang explains that Turing's analysis even has ontological significance, in that "Turing machines are an important piece of evidence for Gödel's belief that sharp concepts exist and that we are capable of perceiving them clearly." 123

The sharp concept is there all along, only we did not perceive it clearly at first. This is similar to our perception of an animal far

with at most a recursively computable provability predicate. This rules out many of the purely semantically defined formalisms such as (full) second order logic with the semantic consequence relation; accordingly our notion of formal system is more general.

${ }^{120}$ op cit.

${ }^{121} \mathrm{Or}$ as Sieg put it in his [78], what was groundbreaking about Turing's analysis was that "Turing solved the Entscheidungsproblem in a way that did full justice to the normative demand for intersubjectivity between humans."

${ }^{122}$ [85], p. 203. Italics ours.

${ }^{123}$ ibid, p. 194. 
away and then nearby. We had not perceived the sharp concept of mechanical procedure sharply before Turing, who brought us to the right perspective. And then we do perceive clearly the sharp concept. ${ }^{124}$

The concept of a Turing machine is formalism free, in our sense of the term, while at the same time it is a concept from which the formal notion can be easily recovered. A Turing machine is neither a formal nor an informal concept, however; or, one could say, it is, in some strange sense, both ${ }^{125}$ - the one thing, the missing piece which anchors the picture.

This rendering of the correspondence between the informal notion of computability and the various formal notions which had been introduced, effectuated via a "perfect" analysis of the informal concept, a correspondence Robin Gandy described as a theorem in his [28], is what Gödel is asking for in his 1946 lecture - not as applied to computability of course, as Turing had already done that. What is needed is to transfer the entire Turing analysis to the "other" epistemological cases, namely definability and provability.

5.2. Provability. We now turn to Gödel's suggestion that something analogous to Turing's analysis of computability is to be expected for the other epistemological notions, namely provability and definability:

This, I think, should encourage one to expect the same thing to be possible also in other cases (such as demonstrability or definability). It is true that for these other cases there exist certain negative results, such as the incompleteness of every formalism ... But close examination shows that these results do not make a definition of the absolute notions concerned impossible under all circumstances, but only exclude certain ways of defining them, or at least, that certain very closely related concepts may be definable in an absolute sense. ${ }^{126}$

We briefly consider Gödel's suggestions regarding provability, before turning to definability, our main concern in this paper.

Let us consider, e.g., the concept of demonstrability. It is well known that, in whichever way you make it precise by means of a formalism, the contemplation of this very formalism gives rise to new axioms which are exactly as evident and justified as those with which you started, and that this process of extension can be extended into the transfinite. So there cannot exist any formalism which would embrace all these steps; but this does not exclude

\footnotetext{
${ }^{124}$ ibid, p. 205.

${ }^{125}$ As Juliet Floyd puts it in [24], "A Turing machine lends itself, intentionally and conceptually, to a double point of view: it is both a formal system and a remodeling."

${ }^{126}[31]$, p. 150 .
} 
that all these steps ... could be described and collected in some non-constructive way. In set theory, e.g., the successive extensions can be most conveniently be represented by stronger and stronger axioms of infinity. It is certainly impossible to give a combinational and decidable characterization of what an axiom of infinity is; but there might exist, e.g., a characterization of the following sort: An axiom of infinity is a proposition which has a certain (decidable) formal structure and which in addition is true. Such a concept of demonstrability might have the required closure property, i.e., the following could be true: Any proof for a set-theoretic axiom in the next higher system above set theory (i.e., any proof involving the concept of truth which I just used) is replaceable by a proof from such an axiom of infinity. It is not impossible that for such a concept of demonstrability some completeness theorem would hold which would say that every proposition expressible in set theory is decidable from the present [ZFC] axioms plus some true assertion about the largeness of the universe of all sets. ${ }^{127}$

In brief, some suitable hierarchy of large cardinal assumptions should replace the hierarchy of formal systems generated by, e.g., the addition of consistency statements to set theory, i.e., passing from ZFC to ZFC+Con(ZFC) and then iterating this; or the addition of a satisfaction predicate for the language of set theory, then considering set theory in the extended language, and iterating this.

The usefulness of transfinite concepts was mentioned by Gödel in a number of contexts, e.g., that they complement partial proofs of the Completeness Theorem given by Löwenheim and Skolem, ${ }^{128}$ while in the case of the Incompleteness Theorem, the statement of the result itself involves transfinite concepts such as "truth." The very prescient statement that the transfinite also assists decidability in set theory, incompleteness being per se a property of formal systems, which can be transcended by means of infinitary concepts, is made here in an embryonic form of what came to be known as Gödel's program for large cardinals, a program laid out in its fullest form in his 1947 "What is Cantor's Continuum Hypothesis?"129

A partial realization of this program to replace logical hierarchies by infinitary principles, and thereby secure decidability, can be seen in the following result of Woodin: in the presence of large cardinals, ${ }^{130}$ the $\Sigma_{1}^{2}$ theory of real numbers, i.e., existential statements about sets of reals, is

\footnotetext{
${ }^{127}[31$, p. 151]

${ }^{128}$ See Gödel/Wang correspondence in [38], e.g., letter on p. 404.

${ }^{129}$ See [32]. In fact very few large cardinals had been discovered in 1946.

${ }^{130} \mathrm{~A}$ proper class of Woodin cardinals.
} 
(set) forcing immune in the sense that their truth cannot be changed by forcing - one important form of decidability. ${ }^{131}$

Of course, forcing extensions do not form the kind of (linear) hierarchy that Gödel has in mind here, 1946 being well before the invention of forcing, never mind the appearance of generic absoluteness theorems. ${ }^{132}$ It would have been very natural for Gödel to have eventually specialized his program to forcing extensions, though, given his overall interest in deciding independent statements such as the Continuum Hypothesis, as we have argued elsewhere.

Is the concept of provability behind these results formalism free in the sense of the other examples given above? Forcing extensions do not form a linear hierarchy but a partial order. An $\Omega$-proof, a proof concept which occurs in Woodin's later work in connection with generic absoluteness, ${ }^{133}$ is just a universally Baire set of reals. Replacing the concept of a proof by a universally Baire set of reals has an appearance of formalism freeness, somewhat reminiscent of the idea of replacing a formula by a set invariant under automorphisms in the AEC context. Universally Baire sets of reals are also very regular in a precise sense. ${ }^{134}$

5.3. Definability. Gödel now passes to definability, in connection with which he can give us "somewhat more definite suggestions."

The idea, again, is to treat the notion of definability in an absolute fashion. Such a goal seems paradoxical, as definability appears to be rather closely tied to a formalism, in the sense that one uses a signature and formation rules to build up well-formed formulas, and then the definable sets are simply declared to be those sets that are definable via that stock of formulas. But the standard technical definition of definability is clearly not what Gödel has in mind. For Gödel, definability is an epistemological notion. Finding an absolute characterization of it is, Gödel says, to find "an adequate formulation for comprehensibility by our mind." 135 Simply laying down a language and then declaring the sets "comprehensible by our mind" to be those given by the formulas of the language, not only introduces arbitrariness - it leads, as Gödel will later observe, ${ }^{136}$ to paradox.

After these initial remarks Gödel introduces the concept of "ordinal definability." 137 The idea is to take the ordinals as already given and then define

\footnotetext{
${ }^{131}$ See [87]. The result requires the Continuum Hypothesis. Another result of this kind Woodin which says about the structure $L(\mathbb{R})$, the constructible closure of the reals, that its first order theory is (set) forcing absolute in the presence of large cardinals (a proper class of Woodin cardinals), [86].

${ }^{132}$ Cohen invented forcing in 1963.

${ }^{133}$ [87].

${ }^{134}$ This point bears elaboration. The reader is referred to the author's subsequent monograph.

${ }^{135}$ [35, p. 152].

${ }^{136}$ to Wang, citing the history of Frege's Axiom V, see [85].

${ }^{137}$ not a concept of definability in his more general sense, see p. 151, [31].
} 
sets by means of the language of set theory as usual, but with finitely many ordinals as parameters.

Here you also have, corresponding to the transfinite hierarchy of formal systems, a transfinite hierarchy of concepts of definability. Again it is not possible to collect together all these languages in one, as long as you have a finitistic concept of language, i.e., as long as you require that a language must have a finite number of primitive terms. But, if you drop this condition, it does become possible ... by means of a language which has as many primitive terms as you wish to consider steps in this hierarchy of languages, i.e., as many as there are ordinal numbers. The simplest way of doing it is to take the ordinals themselves as primitive terms. So one is led to the concept of definability in terms of ordinals ... This concept should, I think be investigated.

The advantage of doing things this way is that ordinals bequeath their "lawlikeness" to the sets constructed from them, namely the ordinal definable sets. They are "formed according to a law." 138

Ordinal definability is itself definable in set theory—something Gödel must have known, judging from his remarks here: "By introducing the notion of truth for this whole transfinite language, i.e., by going over to the next language, you will obtain no new definable sets (although you will obtain new definable properties of sets)"- though modern proofs of this depend on the Levy Reflection Principle, which was only proved in 1960. For this reason, if one passes to the "next language," i.e., one obtained by adding a truth predicate for statements about ordinal definable sets, one obtains no new ordinal definable sets. ${ }^{139}$ Another corollary of the definability of the concept of ordinal definability, in analogy with the computable functions, the notion of ordinal definability is "non-diagonalizable" as well.

In what sense is ordinal definability formalism independent? In fact similar to constructibility, by a result of [84] the class of hereditarily ordinal definable sets can be obtained as the closure of the class whose the elements are all sets of the form $V_{\alpha}$ under the Gödel operations, instead of via the first order language. ${ }^{140}$ This is an apparently formalism free construction, relative to set theory.

What Gödel had in mind though was something different. His goal in these remarks involves replacing a formalism, or more precisely a hierarchy of them generated by the addition of truth predicates, by an axiom or principle of infinity of a special kind: the characterization of the principle must be decidable, and the principle must be true. The principle which is implicit in

\footnotetext{
${ }^{138}[35$, p. 152$]$.

${ }^{139}$ More exactly, for any formula $\phi(x)$ there is another formula $\phi^{\prime}(x)$ which says that $\phi(x)$ is true in HOD. So no new ordinal definable sets are created by referring to truth in HOD.

${ }^{140}[66]$. Here $V_{0}=\emptyset, V_{\alpha+1}=\mathcal{P}\left(V_{\alpha}\right)$ and $V_{v}=\cup_{\alpha<v} V_{\alpha}$ for limit $v$.
} 
the concept of ordinal definability ${ }^{141}$ - the Levy Reflection Principle ${ }^{142}$ would satisfy both requirements for Gödel. We noted above that Gödel does not state this principle in the lecture; but this is the principle that is used in modern accounts to show that HOD is a definable class. The Levy Reflection Principle is not itself an axiom of infinity per se, in fact it is provable; but if it is slightly strengthened and then reflected to some $V_{\alpha}$, it becomes an axiom of infinity. ${ }^{143}$

Gödel next takes up the idea of capturing a different notion of definability in a formalism independent, lawlike and definable way. The presentation of the constructible hierarchy in terms of definability was given by Gödel in 1939 in his monograph on the consistency of the continuum hypothesis; in 1940 he gave a second presentation of the constructible sets, as the closure of the class of ordinals under the so-called "Gödel operations." This latter presentation is somewhat logic or formalism free, as we noted above, in that no satisfaction or definability predicates occur in it. ${ }^{144}$ But Gödel does not see $L$ as exemplifying an, in his sense, absolute notion of definability, even so that, as in the case of ordinal definability, the constructible hierarchy is non-diagonalizable in the following sense: if we form the constructible hierarchy and then add to the language of set theory a predicate for " $x$ is constructible," we do not obtain any new constructible sets.

... but, comparing constructibility with the concept of ordinal definability just outlined, you will find that not all logical means of definition are admitted in the definition of constructible sets. ... This has the consequence that you can actually define sets, and even sets of integers, for which you cannot prove that they are constructible (although this can of course be consistently assumed.) For this reason, I think constructibility cannot be considered a satisfactory formulation of definability. ${ }^{145}$

We will return to constructibility in section 5.1. It turns out that the constructible hierarchy is very robust and permits interesting generalizations.

At the end of the address Gödel remarks of his two candidates for the concept of absolute definability - constructibility and ordinal definabilitythat neither of these is an absolute notion in the sense of the paper:

... in both examples I gave, [ordinal definability and constructibil-

ity] the concepts arrived at or envisaged were not absolute in the strictest sense, but only with respect to a certain system of things,

\footnotetext{
${ }^{141}$ actually HOD here.

${ }^{142}$ The principle says that for every $n$ there are arbitrarily large ordinals $\alpha$ such that $V_{\alpha} \prec_{n} V$.

${ }^{143}$ There is an $\alpha$ such that for all $A \subseteq \alpha$ there is $\beta<\alpha$ with $\left(V_{\beta}, \in, A \cap V_{\beta}\right) \prec\left(V_{\alpha}, \in, A\right)$. This implies that $\alpha$ is (strongly) inaccessible [44, Proposition 6.2.].

${ }^{144} \mathrm{The}$ second, syntactic consistency proof of the $\mathrm{CH}$ is perhaps less perspicuous than the initial proof.

${ }^{145}$ Italics ours. [35], p. 152.
} 
namely the sets as conceived in axiomatic set theory; i.e., although there exist proofs and definitions not falling under these concepts, these definitions and proofs give, or are to give, nothing new within the domain of sets and propositions expressible in terms of "set," " $\epsilon$, , and the logical constants. ${ }^{146}$

It would seem that, for Gödel, an absolute notion of definability "in the strictest sense" would have to dispense altogether with the background theory - set theory, in this case. This is slightly paradoxical in that on the one hand, Gödel's use of the word "absolute" seems to indicate a desire for a characterization of definability which is not tied to the background theoryin this case ZFC set theory; while on the other hand he is clearly reluctant to attach a transcendental concept, i.e., one not definable in set theory, to the "epistemological" notion in question. So while aspiring to absoluteness in the "strictest sense," 147 Gödel is, at the end of the day, committed to set theory as his metatheory. Idealization is decisive here, Gödel will say later, ${ }^{148}$ in connection with the constructible hierarchy. But just as with constructibility, idealization does not have to bring full transcendence.

That this is the fundamental point of tension here bears repeating: Gödel aspires toward absoluteness in the strictest sense, that is to say we read Gödel as taking as his metatheory set theory in the informal sense. "The intuitive notion of the cumulative type structure," as Kreisel puts it in his [53], is the basic standpoint. While on the other hand the only measure of absoluteness - our compass, so to speak - involves, in this case at least, the ZFC formalization.

§6. Implementation. Up to now we have discussed the meaning of the expression "formalism free" in somewhat general terms. We now give one possible sense of the term. Inspired by Gödel's call for extending formalism independence to the concept of definability, we extrapolate to a possible implementation, extending the idea of a Church-Turing Thesis to the notion of definability in the context of set theory.

We begin by fixing a notion of definability, in this case constructibility, and view it as an operator on logics. ${ }^{149}$ We denote the result of applying this operator to a logic $\mathcal{L}^{*}$ by $L\left(\mathcal{L}^{*}\right)$, defined as follows:

$$
\begin{aligned}
L_{\alpha+1}\left(\mathcal{L}^{*}\right)= & \left\{X \subseteq L_{\alpha}\left(\mathcal{L}^{*}\right): X \text { is } \mathcal{L}^{*}\right. \text {-definable } \\
& \left.\quad \text { over }\left(L_{\alpha}\left(\mathcal{L}^{*}\right), \epsilon\right) \text { with parameters. }\right\}, \\
L_{v}\left(\mathcal{L}^{*}\right)= & \bigcup_{\alpha<v} L_{\alpha}\left(\mathcal{L}^{*}\right) \text { for limit } v, \\
L\left(\mathcal{L}^{*}\right)= & \bigcup_{\alpha} L_{\alpha}\left(\mathcal{L}^{*}\right) .
\end{aligned}
$$

\footnotetext{
${ }^{146}$ ibid.

${ }^{147}$ as does Tarski, as we noted above.

${ }^{148}$ to Wang, [85], remark 8.3.3.

${ }^{149}$ See Section 3 for our definition of a logic.
} 
If $\mathcal{L}^{*}$ is taken to be first order logic, denoted $\mathcal{F O}$, we obtain the constructible hierarchy itself. We noted above that Myhill and Scott showed in their [66], that if $\mathcal{L}^{*}$ is taken to be second order logic, denoted $\mathcal{S O}$, the class obtained is HOD, the hereditarily ordinal definable sets. ${ }^{150}$

For another precedent, the so-called Chang model $L\left(\mathcal{L}_{\omega_{1} \omega_{1}}\right)$ is a model of ZF together with the failure of the Axiom of Choice, under large cardinal assumptions. In the light of this failure one might advocate the use of fragments of $\mathcal{S O}$, also on the basis of these having a somewhat more reasonable syntax.

It is not difficult to see that if $\mathcal{L}^{*}$ is taken to be weak second order logic $\mathcal{L}_{w}^{2}$, i.e., the logic allowing quantification over finite sets, we again obtain the constructible hierarchy $L$, that is, $L(\mathcal{F O})=L\left(\mathcal{L}_{w}^{2}\right)=L$. This is interesting, since this represents a change of logic. In particular, $\mathcal{L}_{w}^{2}$ is non-compact.

This brings up an important issue with respect to the question whether the invariance of a concept of definability with respect to a particular class of logics represents the fulfillment of a Church-Turing Thesis, in our sense of the term, for definability. What is meant here by a "change of logic"? For now we will adopt the principle that this is indicated by the failure of properties mentioned in Lindström Theorems, i.e., the failure of compactness and/or the Löwenheim-Skolem Theorem in the relevant cases where such characterizations of logics exist (even partially). ${ }^{151}$ However one may not always have such a measure of change of logic.

Continuing with our observations about $L$-invariance, it is also not difficult to see that if one takes $\mathcal{L}^{*}$ to be that obtained from first order logic by adding to it the quantifiers $Q_{\alpha}$, i.e., "there are at least $\aleph_{\alpha}$ many," for all cardinals $\aleph_{\alpha}$, one again obtains $L .{ }^{152}$ Our eventual focus is on fragments of second order logic, and indeed when only cardinals that are second order characterizable are used, this logic is a fragment of second order logic. ${ }^{153}$

The following implementation of Gödel's suggestion in the 1946 lecture emerges. Define the equivalence relation on logics:

$$
\mathcal{L}^{*} \equiv \mathcal{L}^{* *} \text { if and only if } L\left(\mathcal{L}^{*}\right)=L\left(\mathcal{L}^{* *}\right) .
$$

This equivalence relation partitions the family of all logics into classes inside which $L$ is indifferent to what logic is used. Conceivably the equivalence classes could be rather small. We would interpret this by saying that

\footnotetext{
${ }^{150}$ In fact this result enjoys some robustness, i.e., ostensibly much weaker logics than second order logic still give rise to HOD. See [50].

${ }^{151}$ We note here that for constructive logic there are classical, i.e., not constructively valid, proofs of compactness and Löwenheim-Skolem.

${ }^{152}$ See [50].

${ }^{153} \mathrm{~A}$ cardinal $\kappa$ is second order characterizable if there is a second order sentence $\phi$ of the empty vocabulary such that for all models $M$ of the empty vocabulary, $M$ has cardinality $\kappa$ iff $M \models \phi$. All the $\aleph_{n}{ }^{\prime} s$ are second order characterizable. If $\kappa$ is second order characterizable, then so are $\kappa^{+} 2^{\kappa}, 2^{2^{\kappa}}$, etc. See [81].
} 
$L$ seems to be quite dependent on the formalism used. As it happens, the classes are big, the class of $\mathcal{F O}$ including at least $\mathcal{L}_{w}^{2}$, i.e., the logic allowing quantification over finite sets, the family of extensions of $\mathcal{F O}$ given by $\mathcal{L}\left(Q_{\alpha}\right)$ for each $\aleph_{\alpha}$, and finally Magidor-Malitz logic, assuming $0^{\sharp}$ exists. ${ }^{154}$ $L$ in that sense "reads" all of these logics, including first order logic with the Magidor-Malitz quantifier adjoined to it, as first order.

In fact, more is true: ${ }^{155}$ The constructible hierarchy $L$ is unaffected if first order logic is enriched in the construction of $L$ by any of the following, simultaneously or separately:

- Recursive infinite conjunctions $\bigwedge_{n=0}^{\infty} \phi_{n}$ and disjunctions $\bigvee_{n=0}^{\infty} \phi_{n}$.

- Cardinality quantifiers $Q_{\alpha}, \alpha \in O n$.

- Equivalence quantifiers ${ }^{156} Q_{\alpha}^{E}, \alpha \in O n$.

- Well-ordering quantifier

$$
\begin{aligned}
\mathcal{M} F W x, y \phi(x, y) \Longleftrightarrow & \\
& \left\{(a, b) \in M^{2}: \mathcal{M} \models \phi(a, b)\right\} \text { is a well-ordering. }
\end{aligned}
$$

- Recursive game quantifiers

$$
\begin{aligned}
& \forall x_{0} \exists y_{0} \forall x_{1} \exists y_{1} \ldots \bigwedge_{n=0}^{\infty} \phi_{n}\left(x_{0}, y_{0}, \ldots, x_{n}, y_{n}\right), \\
& \forall x_{0} \exists y_{0} \forall x_{1} \exists y_{1} \ldots \bigvee_{n=0}^{\infty} \phi_{n}\left(x_{0}, y_{0}, \ldots, x_{n}, y_{n}\right) .
\end{aligned}
$$

We suggest that this manifests a remarkable independence of $L$ from the formalism used, and in that sense provides evidence for Gödel's suggestion that constructibility might be a good candidate for a formalism independent notion of definability - though not in the way he imagined it at the time, evidently. Constructibility being not particularly sensitive to the underlying logic in that sense gives evidence that a type of Church-Turing thesis holds for $L$, namely invariance with respect to a certain large class of logics.

We can also change our point of view and consider the equivalence of a logic in the above equivalence relation as a measure of similarity of these logics. Thus logics which are in this sense equivalent to first order logic are then considered "similar to first order logic, as far as constructibility

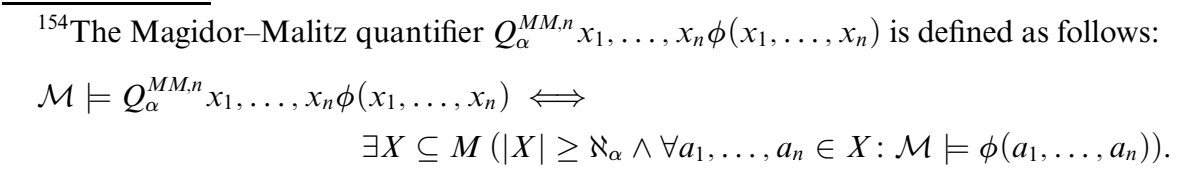

By a result of Magidor, if $\mathcal{L}^{*}$ is taken to be first order logic with the Magidor-Malitz quantifiers adjoined to it, then also then $\mathcal{L}^{*} \equiv \mathcal{F O}$, assuming $0^{\sharp}$ exists. [50].

${ }^{155}$ See [50] for details.

${ }^{156}$ These are quantifiers which say that a given definable equivalence relation has $\aleph_{\alpha}$ equivalence classes. 
is concerned." That is to say, $L$ "reads" the logics in the class as first order. Respectively, logics which are in this sense equivalent to second order logic are then considered "similar to second order logic, as far as constructibility is concerned." Work on this is underway but already quite surprising connections between logics have emerged, connections that other measures of similarity between logics have not revealed. ${ }^{157}$

In the above results we considered certain fragments of second order logic. Why are these logics interesting? Second order logic has emerged in recent decades in connection with structuralism and other foundational issues. One would think that the study of its fragments, especially those which are (countably) compact and have completeness theorems ${ }^{158}$ or other desirable properties, might also be of interest. That is, rather than look at a logic so strong that it has no proof concept, one might want to investigate the territory between first and second order logic; of, in this case, $L$ up to HOD, where HOD is taken in the sense of $L(\mathcal{S O})$, the version of $L$ obtained by replacing the underlying first order logic in the construction of $L$ with second order logic.

6.1. Beyond $L$. In fact the restriction to $L$ is not intrinsic to our analysis. We mentioned that also HOD is not particularly sensitive to the underlying logic and indeed one can apply a concept $D$ of definability to any suitable logic in the operation $\mathcal{L}^{*} \mapsto D\left(\mathcal{L}^{*}\right)$, and design a Church-Turing Thesis of this this sort, relative to $D$.

The version of formalism freeness considered here involves formalism or more precisely logic independence, i.e., invariance under substitution of one of a class of logics, considered on a case by case basis. In the case of definability this may not be the only choice. One could imagine other aspects of a formalism with respect to which a given concept of definability might stabilize. However there are no obvious candidates on the horizon.

Thinking beyond definability toward other canonical concepts, one might also consider this schema, i.e., varying the underlying logic, in other contexts. In fact any logical hierarchy, e.g., Kleene's ramified hierarchy of reals ${ }^{159}$ is amenable to this treatment, conceivably.

6.2. Extending the axioms of set theory. Instead of considering a particular canonical set-theoretic construction, we can ask, does the theory ZFC itself, or any of its semantic extensions, admit a Church-Turing Thesis of the kind we have been considering? There are a number of ways of answering the question. Our approach is the following: given a logic $\mathcal{L}^{*}$, exchange $\mathcal{F O}$ in the Separation and Replacement Axioms of ZFC with another logic $\mathcal{L}^{*}$, obtaining an $\mathcal{L}^{*}$-version of $\mathrm{ZFC}$, denoted $\mathrm{ZFC}\left(\mathcal{L}^{*}\right)$. More exactly, the

\footnotetext{
${ }^{157}$ See [50].

${ }^{158}$ See [47].

${ }^{159}[51]$.
} 
modification is that the formula $\phi(x, \vec{y})$ in the Schema of Separation

$$
\forall x \forall x_{1} \ldots \forall x_{n} \exists y \forall z(z \in y \leftrightarrow(z \in x \wedge \phi(z, \vec{x})))
$$

and the formula $\psi(u, z, \vec{x})$ in the Schema of Replacement

$$
\begin{aligned}
\forall x \forall x_{1} \ldots \forall x_{n}\left(\forall u \forall z \forall z^{\prime}((u \in x\right. & \left.\left.\wedge \psi(u, z, \vec{x}) \wedge \psi\left(u, z^{\prime}, \vec{x}\right)\right) \rightarrow z=z^{\prime}\right) \\
\rightarrow & \exists y \forall z(z \in y \leftrightarrow \exists u(u \in x \wedge \psi(u, z, \vec{x})))) .
\end{aligned}
$$

are allowed to be taken from $\mathcal{L}^{*}$ rather than just $\mathcal{F O}$. We do not add logical rules for $\mathcal{L}^{*}$-formulas, rather the logic $\mathcal{L}^{*}$ is semantically defined, the concept of a model $(M, E), E \subseteq M \times M$, satisfying the axioms $\mathrm{ZFC}\left(\mathcal{L}^{*}\right)$ being obviously well-defined. Our question is, to what extent is $\mathrm{ZFC}\left(\mathcal{L}^{*}\right)$ dependent on $\mathcal{L}^{*}$ ?

Note that $\mathrm{ZFC}\left(\mathcal{L}^{*}\right)$ is at least as strong as $\mathrm{ZFC}$ in the sense that every model of $\mathrm{ZFC}\left(\mathcal{L}^{*}\right)$ is, a fortiori, a model of ZFC. The class of models of $\mathrm{ZFC}$ is immensely rich, ZFC being a first order theory. We now ask the question, what can we say about the models of $\mathrm{ZFC}\left(\mathcal{L}^{*}\right)$ for various logics $\mathcal{L}^{*}$ ?

If we think of the equivalence classes of logics defined by

$$
\mathcal{L}^{*} \equiv \mathcal{L}^{* *} \text { if and only if } \operatorname{ZFC}\left(\mathcal{L}^{*}\right)=\operatorname{ZFC}\left(\mathcal{L}^{* *}\right)
$$

the following is true: The class of $\mathcal{L}\left(Q_{0}\right)$ contains all logics that can express "finiteness" and are eliminable ${ }^{160}$ in $\omega$-models of set theory, for example weak second order logic. The class of $\mathcal{L}\left(Q_{0}^{M M}\right)$ contains all logics capable of expressing well-foundedness, which are eliminable in transitive models of set theory, such as strictly absolute logics, e.g., the recursive game quantifier. Finally, the class of $\mathcal{L}_{\omega_{1} \omega}$ contains all logics between $\mathcal{L}_{\omega_{1} \omega}$ and $\mathcal{L}_{\omega_{1} \omega_{1}}$. See [50] for details.

So similar to the case of $L\left(\mathcal{L}^{*}\right)$, the class of models of $\operatorname{ZFC}\left(\mathcal{L}^{*}\right)$ is somewhat dependent on the choice of $\mathcal{L}^{*}$, while at the same time there is also a great deal of variability as to the choice of $\mathcal{L}^{*}$. Our working hypothesis in this area-our expansive intuitionism, as it were-is that $\mathrm{ZFC}\left(\mathcal{L}^{*}\right)$ has semantic content independently of $\mathcal{L}^{*}$, short of very dramatic variations in the strength of $\mathcal{L}^{*}{ }^{161}$

The topic of semantic extensions of ZFC, as well as the related topic of set theory and arithmetic in extensions of first order logic, are little studied and full of open problems.

\footnotetext{
${ }^{160}$ i.e., can be first order defined.

${ }^{161}$ Other extensions of this kind have been considered. In his [45] M. Kaufmann added the stationary quantifier (see [9]) to ZFC together with some natural axioms concerning the stationary quantifier, and proved in the new system, among other things, the consistency of ZFC. See also A. MacIntyre's [59], and Schmerl and Simpson's [72].
} 
$\S 7$. Concluding remarks. Are mathematical objects "language-embodied" in some essential way? Are mathematical objects presented, or grasped, or perceived - however one wants to put it —only insofar as they are embedded in a language? In a formal language? Or does our mathematical discourse have an autonomously given, formalism independent content, in the sense given in Section 4 of this paper, or in any other sense?

A thesis which is close to the heart of the naturalist, at least of the methodological, second philosophical kind we considered earlier in this paper, is that the question of meaning in mathematics is not amenable to frameworks of analysis originating in the a priorist philosophical tradition. "First philosophical" theorizing about meaning, to use Maddy's terminology, that is, theorizing articulated from within and beholden to standards external to mathematical practice, standards which are judged to have been met-or not - according to extra-mathematical criteria, even insofar as such criteria originate in natural science ${ }^{162}$; that philosophical undertaking, is, for the naturalist, to be discouraged - greatly. ${ }^{163}$

The view taken here is that the second philosopher gets something right about meaning. At the same time, though, meaning does show up for us from time to time, provided we look for it in the right way. Alva Noë writes: "the world shows up for us." 164 Noë is alluding to the fact that our experience of the world is direct and incorrigible, rather than mediated, either by concepts, or representations, or relations between these, etc.:

Frege claimed that statements of number are statements about concepts $(1884 / 1978,59)$. The statement "the King's carriage is drawn by four horses," for example, is a statement about the concept "horse that draws the King's carriage." It might look as if we are talking about the King's carriage, when we use these words, but we aren't ...

Noe concedes that Frege's analysis of the situation is reasonable, as far as it goes; nevertheless he insists that when we speak of the King's carriage being drawn by four horses, we are speaking not about the concept of "horse" or the concept of "four"; rather we are saying something about horses and the number four, so to say, directly.

To say that mathematics is, and has always been, practiced in a formalism free way is just to say that, in mathematics, the world shows up for us. More precisely, we can provide something in the way of evidence for the entanglement - or the lack of it - of our natural mathematical discourse, not only with the (formalized) concept of number but with an entire formal apparatus. This is because the beautiful field of mathematical logic has developed to that point.

\footnotetext{
${ }^{162}$ See Maddy, [1997, 184].

${ }^{163}$ E.g., this was Quine's view of meaning, at least prior to his 1970 Philosophy of Logic.

${ }^{164}$ Varieties of Presence, [67].
} 
At the beginning of this paper we mentioned the problem of consistency. Formalisms are one particular way that mathematicians represent the mathematical field to themselves. As a "coping tool" they are essential in just the way that art is essential - they tell us where we are. But Gödel put his finger on the main issue in the opening remarks of his Dialectica paper: for a proof of consistency one is forced to deal directly with meaning-mere reflection on the combinatorial properties of syntax will never lead to a consistency proof.

How fortunate we are then, that meaning is right there.

\section{REFERENCES}

[1] Hans AdLer, Thorn-forking as local forking, Journal of Mathematical Logic, vol. 9 (2009), no. 1, pp. 21-38.

[2] Andrew Arana and Paolo Mancosu, On the relationship between plane and solid geometry, Review of Symbolic Logic, vol. 5 (2012), no. 2, pp. 294-353.

[3] John T. Baldwin, Formalization, primitive concepts, and purity, The Review of Symbolic Logic, vol. 6 (2013), no. 2, pp. 87-128.

[4] - Completeness and categoricity (in power): Formalization without foundationalism, preprint.

[5] John T. Baldwin, Tapani Hyttinen, and Meeri Kesälä, Beyond first order logic: From number of structures to structure of numbers part I, Bulletin of the Iranian Mathematical Society, vol. 39 (2013), no. 1, pp. 1-26.

[6] - Beyond first order logic: From number of structures to structure of numbers part II, Bulletin of the Iranian Mathematical Society, vol. 39 (2013), no. 1, pp. 27-48.

[7] JON BARWISE, Absolute logics and $L_{\infty \omega}$, Annals of Mathematical Logic, vol. 4 (1972), pp. $309-340$.

[8] - Axioms for abstract model theory, Annals of Mathematical Logic, vol. 7 (1974), pp. $221-265$.

[9] Jon Barwise, Matt Kaufmann, and Michael Makkai, Stationary logic, Annals of Mathematical Logic, vol. 13 (1978), no. 2, pp. 171-224.

[10] ERRETt Bishop, Foundations of constructive analysis, McGraw-Hill Book Co., 1967.

[11] Nicholas Bourbaki, The architecture of mathematics, The American Mathematical Monthly, vol. 57 (1950), pp. 221-232.

[12] L. E. J. BRouwEr, Brouwer's Cambridge lectures on intuitionism, Cambridge University Press, Cambridge, 1981, edited by D. van Dalen.

[13] JoHn Burgess, Putting structuralism in its place, retrieved from www . princeton . edu/ $\sim$ jburgess/anecdota.htm, 2009.

[14] Samuel R. Buss, Alexander S. Kechris, Anand Pillay, and Richard A. Shore, The prospects for mathematical logic in the twenty-first century, this BULLETIN, vol. 7 (2001), no. 2, pp. 169-196.

[15] Martin Davis, Computability and unsolvability, McGraw-Hill Series in Information Processing and Computers, McGraw-Hill Book Co., 1958.

[16] Martin Davis (editor), The undecidable, Dover Publications Inc., Mineola, NY, 2004, corrected reprint of the 1965 original [Raven Press, Hewlett, NY].

[17] Michael Detlefsen, Poincaré vs. Russell on the rôle of logic in mathematics, Philosophia Mathematica. Series III, vol. 1 (1993), no. 1, pp. 24-49.

[18] — What does Gödel's second theorem say?, Philosophia Mathematica. Series III, vol. 9 (2001), no. 1, pp. 37-71, The George Boolos Memorial Symposium, II. 
[19] Michael Detlefsen and Andrew Arana, Purity of methods, Philosophers' Imprint, vol. 11 (2011), no. 2, pp. 1-20.

[20] M. A. Dickmann, Large infinitary languages, Studies in Logic and the Foundations of Mathematics, vol. 83, North-Holland, 1975.

[21] H.-D. EbBinghaus, Extended logics: the general framework, Model-theoretic logics, Perspectives in Mathematical Logic, Springer, 1985, pp. 25-76.

[22] Ronald Fagin, Probabilities on finite models, The Journal of Symbolic Logic, vol. 41 (1976), no. 1, pp. 50-58.

[23] Solomon Feferman, Arithmetization of metamathematics in a general setting, Polska Akademia Nauk. Fundamenta Mathematicae, vol. 49 (1960/1961), pp. 35-92.

[24] Juliet Floyd, The varieties of rigorous experience, The Oxford handbook of the history of analytic philosophy (M. Beaney, editor), Oxford Handbooks in Philosophy, Oxford University Press, Oxford, 2013.

[25] Abraham A. Fraenkel and Yehoshua Bar-Hillel, Foundations of set theory, Studies in Logic and the Foundations of Mathematics, North-Holland, 1958.

[26] CURTIS Franks, The autonomy of mathematical knowledge, Cambridge University Press, Cambridge, 2009.

[27] - Logical completeness, form, and content: an archaeology, Interpreting Gödel (J. Kennedy, editor), Cambridge University Press, Cambridge, to appear.

[28] Robin Gandy, The confluence of ideas in 1936, The universal Turing machine: a half-century survey, Oxford Science Publications, Oxford University Press, 1988, pp. 55-111.

[29] KURT GöDEL, Die Vollständigkeit der Axiome des logischen Funktionenkalküls, Monatshefte für die Mathematik, vol. 37 (1930), pp. 349-360.

[30] - The Consistency of the Continuum Hypothesis, Annals of Mathematics Studies, no. 3, Princeton University Press, Princeton, NJ, 1940.

[31] — Remarks before the Princeton bicentennial conference of problems in mathematics, 1946, reprinted in Collected Works. II, (S. Feferman et al., editors), Oxford University Press, Oxford, 1990.

[32] - What is Cantor's continuum problem?, The American Mathematical Monthly, vol. 54 (1947), pp. 515-525.

[33] - Über eine bisher noch nicht benützte Erweiterung des finiten Standpunktes, Dialectica, vol. 12 (1958), pp. 280-287.

[34] — Collected Works. I: Publications 1929-1936, (S. Feferman et al., editors), Oxford University Press, Oxford, 1986.

[35] - Collected Works. II: Publications 1938-1974, (S. Feferman et al., editors), Oxford University Press, Oxford, 1990.

[36] - Collected Works. III: Unpublished essays and lectures, (S. Feferman et al., editors), Oxford University Press, Oxford, 1995.

[37] - Collected Works. IV: Correspondence A-G, (S. Feferman et al., editors), Oxford University Press, Oxford, 2003.

[38] - Collected Works. V: Correspondence H-Z, (S. Feferman et al., editors), Oxford University Press, Oxford, 2003.

[39] A. Heyting, Intuitionism: An introduction, second revised ed., North-Holland, 1966.

[40] DaVId HILBERT, Grundlagen der Geometrie, fourteenth ed., Teubner-Archiv zur Mathematik. Supplement, vol. 6, 1999.

[41] WILFRID Hodges, What is a structure theory?, Bulletin of the London Mathematical Society, vol. 19 (1987), pp. 209-237.

[42] Ehud Hrushovski and Boris Zilber, Zariski geometries, Journal of the American Mathematical Society, vol. 9 (1996), no. 1, pp. 1-56. 
[43] TAPANi HytTINEN and HeIKKi TUURI, Constructing strongly equivalent nonisomorphic models for unstable theories, Annals of Pure and Applied Logic, vol. 52 (1991), no. 3, pp. 203248.

[44] Akiniro Kanamori, The higher infinite, second ed., Springer Monographs in Mathematics, Springer, 2009.

[45] Matt Kaufmann, Set theory with a filter quantifier, The Journal of Symbolic Logic, vol. 48 (1983), no. 2, pp. 263-287.

[46] D. KAZHDAN, Lecture notes in motivic integration, available online at www .ma.huji. ac.il/ ${ }^{\text {kazhdan/. }}$

[47] H. Jerome KeISLER, Logic with the quantifier "there exist uncountably many", Annals of Pure and Applied Logic, vol. 1 (1970), pp. 1-93.

[48] JulietTe KenNedy, On embedding models of arithmetic into reduced powers, Matemática Contemporânea, vol. 24 (2003), pp. 91-115, 8th Workshop on Logic, Language, Informations and Computation-WoLLIC'2001.

[49] — Gödel's thesis: An appreciation, Kurt Gödel and the foundations of mathematics (M. Baaz et al., editors), Cambridge University Press, Cambridge, 2011, pp. 95-109.

[50] Juliette Kennedy, Menachem Magidor, and Jouko VäÄnÄnen, Inner models from extended logics, preprint.

[51] S. C. KLEENE, Recursive functionals and quantifiers of finite types. I, Transactions of the American Mathematical Society, vol. 91 (1959), pp. 1-52.

[52] Peter Koepke and Ryan Siders, Register computations on ordinals, Archive for Mathematical Logic, vol. 47 (2008), no. 6, pp. 529-548.

[53] G. KReISEL, Informal rigour and completeness proofs, Proceedings of the International Colloquium in the Philosophy of Science (Imre Lakatos, editor), vol. 1, North-Holland, 1967, pp. 138-157.

[54] - Which number theoretic problems can be solved in recursive progressions on $\Pi_{1}^{1}$-paths through O?, The Journal of Symbolic Logic, vol. 37 (1972), pp. 311-334.

[55] David W. Kueker, Abstract elementary classes and infinitary logics, Annals of Pure and Applied Logic, vol. 156 (2008), no. 2-3, pp. 274-286.

[56] Per Lindström, On extensions of elementary logic, Theoria, vol. 35 (1969), pp. 1-11.

[57] E. G. K. LÓPEZ-EsCOBAR, Introduction, Infinitary logic: in memoriam Carol Karp, Lecture Notes in Mathematics, vol. 492, Springer, Berlin, 1975, pp. 1-16.

[58] N. Luzin, Sur les ensembles projectifs de M. Henri Lebesgue, Comptes Rendus de l'Académie des Sciences. Paris, vol. 180 (1925), no. 2, pp. 1572-1574.

[59] Angus Macintyre, Ramsey quantifiers in arithmetic, Model theory of algebra and arithmetic, Lecture Notes in Mathematics, vol. 834, Springer, Berlin, 1980, pp. 186-210.

[60] Penelope Maddy, Some naturalistic reflections on set theoretic method, Topoi. An International Review of Philosophy, vol. 20 (2001), no. 1, pp. 17-27.

[61] - Second philosophy, Oxford University Press, Oxford, 2009.

[62] - Defending the axioms: on the philosophical foundations of set theory, Oxford University Press, Oxford, 2011.

[63] P. Mancosu, From Brouwer to Hilbert, Oxford University Press, Oxford, 1998.

[64] Kenneth McAloon, Consistency results about ordinal definability, Annals of Pure and Applied Logic, vol. 2 (1970/71), no. 4, pp. 449-467.

[65] Colin McLarty, Poincaré: mathematics \& logic \& intuition, Philosophia Mathematica. Series III, vol. 5 (1997), no. 2, pp. 97-115.

[66] John Myhill and Dana Scott, Ordinal definability, Axiomatic Set Theory, American Mathematical Society, Providence, RI, 1971, pp. 271-278.

[67] Alva NoË, Varieties of presence, Harvard University Press, 2012.

[68] Charles Parsons, Some consequences of the entanglement of logic and mathematics, Reference, rationality, and phenomenology: Themes from Follesdal (Michael Frauchiger, 
editor), Lauener Library of Analytical Philosophy, vol. 2, Ontos Verlag, Frankfurt, 2013, pp. $153-178$.

[69] Anand Pillay and Charles Steinhorn, Definable sets in ordered structures, Bulletin of the American Mathematical Society. New Series, vol. 11 (1984), no. 1, pp. 159-162.

[70] W. V. QuIne, Philosophy of logic, second ed., Harvard University Press, Cambridge, MA, 1986

[71] Abraham Robinson, On the metamathematics of algebra, Studies in Logic and the Foundations of Mathematics, North-Holland, 1951.

[72] JAMEs H. SCHMERL and STEPHEN G. Simpson, On the role of Ramsey quantifiers in first order arithmetic, The Journal of Symbolic Logic, vol. 47 (1982), no. 2, pp. 423-435.

[73] Dana Scott, On constructing models for arithmetic, Infinitistic Methods, Pergamon, Oxford, 1961, pp. 235-255.

[74] Stewart Shapiro (editor), The Oxford handbook of philosophy of mathematics and logic, Oxford Handbooks in Philosophy, Oxford University Press, Oxford, 2005.

[75] Saharon Shelah, Classification Theory for Abstract Elementary Classes, Studies in Logic: Mathematical logic and foundations, vol. 18, College Publications, 2009.

[76] - Classification Theory for Abstract Elementary Classes 2, Studies in Logic: Mathematical Logic and Foundations, vol. 20, College Publications, 2009.

[77] - Nice infinitary logics, Journal of the American Mathematical Society, vol. 25 (2012), no. 2, pp. 395-427.

[78] Wilfried Sieg, Gödel on computability, Philosophia Mathematica. Series III, vol. 14 (2006), pp. 189-207.

[79] W. W. TAIT, Remarks on finitism, Reflections on the foundations of mathematics, Lecture Notes in Logic, vol. 15, Association for Symbolic Logic, Urbana, IL, 2002, pp. 410 419.

[80] Jouko VÄÄNÄNEN, Second-order logic and foundations of mathematics, this BULLETIN, vol. 7 (2001), no. 4, pp. 504-520.

[81] - Second-order logic or set theory?, this BuLLETIN, vol. 18 (2012), no. 1, pp. 91121.

[82] Mark van Atten and Juliette Kennedy, Gödel's modernism: On set-theoretic incompleteness, revisited, Logicism, intuitionism, and formalism, what has become of them? (S. Lindström, E. Palmgren, K. Segerberg, and V. Stoltenberg-Hansen, editors), Synthese Library, vol. 341, Springer, 2009, pp. 303-355.

[83] Lou VAN DEN DRIES, A generalization of the Tarski-Seidenberg theorem, and some nondefinability results, Bulletin of the American Mathematical Society. New Series, vol. 15 (1986), no. 2, pp. 189-193.

[84] P. VopenKa, B. BALCAR, and P. HAJEK, The notion of effective sets and a new proof of the consistency of the axiom of choice (abstract), The Journal of Symbolic Logic, vol. 33 (1968), pp. 495-496.

[85] Hao Wang, A logical journey, Representation and Mind, MIT Press, Cambridge, MA, 1996.

[86] W. Hugh Woodin, Supercompact cardinals, sets of reals, and weakly homogeneous trees, Proceedings of the National Academy of Sciences of the United States of America, vol. 85 (1988), no. 18, pp. 6587-6591.

[87] — The axiom of determinacy, forcing axioms, and the nonstationary ideal, revised ed., de Gruyter Series in Logic and its Applications, vol. 1, Walter de Gruyter, Berlin, 2010.

[88] BorIs ZILBER, A categoricity theorem for quasi-minimal excellent classes, Logic and its applications, Contemporary Mathematics, vol. 380, American Mathematical Society, Providence, RI, 2005, pp. 297-306. 
DEPARTMENT OF MATHEMATICS AND STATISTICS

P.O. BOX 68 (GUSTAF HÄLLSTRÖMIN KATU 2B)

FI-00014 UNIVERSITY OF HELSINKI, FINLAND

E-mail: juliette.kennedy@helsinki.fi 